\title{
THE CONSTRUCTION OF DIRAC OPERATORS ON ORIENTIFOLDS
}

\author{
SIMON KITSON*
}

\begin{abstract}
Motivated by Wigner's theorem, a canonical construction is described that produces an Atiyah-Singer Dirac operator with both unitary and anti-unitary symmetries. This Dirac operator includes the Dirac operator for $K R$-theory as a special case, filling a long-standing gap in the literature. In order to make the construction, orientifold Spin ${ }^{\mathrm{c}}$-structures are defined and classified using semi-equivariant Dixmier-Douady theory, and analogues of several standard theorems on the existence of Spin ${ }^{\mathrm{C}}$-structures are proved.
\end{abstract}

\section{Contents}

1 Introduction 1

2 Orientifolds 3

2.1 Orientifold Groups . . . . . . . . . . . . . . . 4

2.2 Orientifolds . . . . . . . . . . 7

2.3 Orientifold Bundles . . . . . . . . . . . . . 7

2.4 Operations on Orientifold Bundles . . . . . . 10

2.5 Classification of Spinc ${ }^{\mathrm{C}}$-Structures on Orientifolds 12

3 Dirac Operators on Orientifolds 22

3.1 Orientifold Spinor Bundles ........ . . 22

3.2 Connections in Orientifold Spinor Bundles . . 25

3.3 Dirac Operators on Orientifolds . . . . . . . . 28

4 Related Work and Applications 29

A Semi-equivariant Constructions 32

A.1 Semi-equivariance and Associated Bundles . . 32

A.2 Semi-equivariant Connections . . . . . . 33

References $\quad 35$

\section{INTRODUCTION}

This paper uses new results on semi-equivariant Dixmier-Douady theory [21] to determine the orientiation conditions that allow the con-

${ }^{*}$ I would like to thank the Mathematical Sciences Institute of the Australian National University for the postdoctoral fellowship which supported this research. 
struction of Atiyah-Singer Dirac operators with both linear and antilinear symmetries. The construction will be described in detail. The existence of Dirac operators with linear/anti-linear symmetries is basic to the compatibility between index theory and Wigner's Theorem. It also fills the gap in the literature regarding the existence of Dirac operators for $K R$-theory. In addition, it seems likely that such operators have important applications in orientifold string theories and condensed matter physics.

In the present context, the term orientifold will refer to a manifold equipped with an action of a group $\Gamma$ which, in turn, is equipped with a homomorphism $\epsilon: \Gamma \rightarrow \mathbb{Z}_{2}$. This small amount of extra structure is used to define unitary/anti-unitary actions of $\Gamma$ on complex vector bundles over the orientifold. An element $\gamma \in \Gamma$ acts via a unitary map if $\gamma \in \Gamma^{+}:=\operatorname{ker}(\epsilon)$, or an anti-unitary map if $\gamma \in \Gamma^{-}:=\Gamma \backslash \Gamma^{+}$. These vector bundles will be described as orientifold bundles. Note that the set of orientifold bundles over an orientifold depends on the embedding $\Gamma^{+} \hookrightarrow \Gamma$. More generally, the term orientifold will be used as an adjective to describe objects carrying, or compatible with, unitary/anti-unitary actions. For example, the Dirac operator mentioned above acts between orientifold bundles in an equivariant manner and will be described as the orientifold Dirac operator.

The construction of the orientifold Dirac operator depends on an understanding of the global topology of complex vector bundles with anti-unitary symmetries. The main obstacle to understanding the conditions under which an orientifold Dirac operator exists is the failure of equivariant transition cocycles and cohomology to accomodate antilinear symmetries. This obstacle was overcome in [21] by introducing semi-equivariant transition cocycles, which simultaneously generalise Wigner's corepresentations [29, pp. 334-335] [16, pp. 169-172] and equivariant transition cocycles. Using the results of [21], the obstruction to the existence of an orientifold Dirac operator can be identified as a semi-equivariant Dixmier-Douady class. The main results are as follows

(a) Definition 31 defines $\left(\operatorname{Spin}^{\mathrm{c}}, \kappa_{\epsilon}\right)$-structures. These are the appropriate analogue of Spin ${ }^{\mathrm{c}}$-strucutre for orientifolds.

(b) Definition 36 defines the third integral orientifold Stiefel-Whiney class, denoted $W_{3}^{(\Gamma, \epsilon)}$.

(c) Corollary 33 shows that $W_{3}^{(\Gamma, \epsilon)}$ is the obstruction to the existence of a $\left(\operatorname{Spin}^{\mathrm{c}}, \kappa_{\epsilon}\right)$-structure. 
(d) Corollary 34 shows that $\left(\operatorname{Spin}^{\mathrm{c}}, \kappa_{\epsilon}\right)$-structure are classified by $H_{\Gamma}^{2}\left(X,\left(\mathbb{Z}, \iota_{\epsilon}\right)\right)$, the elements of which correspond to semi-equivariant principal $\left(\mathrm{U}(1), \kappa_{\epsilon}\right)$-bundles.

(e) Theorem 41 provides an alternative criteria for the existence of a $\left(\operatorname{Spin}^{\mathrm{c}}, \kappa_{\epsilon}\right)$-structure $P \rightarrow \operatorname{Fr}(V)$ based on the existence of a semiequivariant $\left(\mathrm{U}(1), \kappa_{\epsilon}\right)$-bundle that compliments the equivariant frame bundle $\operatorname{Fr}(V)$.

(f) Definition 54 defines the (Clifford-linear) orientifold Dirac operator and reduced orientifold Dirac operator.

These results yeild the primary theorem of this paper.

Theorem (57). Let $X$ be an orientifold with orientifold group $(\Gamma, \epsilon)$.

(a) If $W_{3}^{(\Gamma, \epsilon)}(X)=0$, then $X$ carries an orientifold Dirac operator.

(b) If $W_{3}^{(\Gamma, \epsilon)}(X)=0$ and $\operatorname{dim}(X)=8$, then $X$ carries a reduced orientifold Dirac operator.

In particular, if $X$ is an 8-dimensional Real manifold and $W_{3}^{\left(\mathbb{Z}_{2}, \mathrm{id}\right)}(X)=0$, then $X$ carries a reduced Real Dirac operator.

These results appeared originally in the authors thesis [20]. The relationship between the constructions described in this paper and other work in the literature will be discussed in $\S 4$, along with some potential applications.

\section{ORIENTIFOldS}

This section begins with a discussion of orientifold groups. Orientifold groups are topological groups equipped with a small amount of extra structure that allows them to act in a linear/anti-linear manner. The representation theory of such actions on finite dimensional complex vector spaces can be reduced to the theory of unitary representations that are invariant under a conjugate structure on the space of equivalence classes of representations. This reduction is achieved by using the Wigner's notion of a corepresentation [29, pp. 334-335] [16, pp. 169-172], which coincides precisely with that of a semi-equivariant $\left(\mathrm{U}(n), \kappa_{\epsilon}\right)$-valued transition cocycle [21, §3] over a point.

After briefly defining orientifolds, orientifold bundles will be introduced as complex vector bundles equipped with linear/anti-linear actions. On any orientifold bundle, it is possible to construct a hermitian metric that is compatible with the linear/anti-linear action. Moreover, 
the frame bundle of an orientifold bundle is a $\Gamma$-semi-equivariant principal $\left(\mathrm{U}(n), \kappa_{\epsilon}\right)$-bundle. Thus, a neat generalisation is formed, in which an orientifold bundle over a point is an orientifold representation, and the semi-equivariant $\left(\mathrm{U}(n), \kappa_{\epsilon}\right)$-valued transition cocycle of its frame bundle is the corresponding corepresentation. From this perspective, the semi-equivariant transition cocycles defined in [21, §3] are generalised corepresentations. As with equivariant bundles, orientifold bundles admit a number of natural operations. Semi-equivariant cocycles again prove useful, in $\S 2.4$, for defining and working with these operations.

2.1. Orientifold Groups. Any group $\Gamma$ which acts by a combination of linear and anti-linear operators must have an index-2 subgroup of elements which act via linear operators, and a complementary subset of elements which act via anti-linear operators. In general, if $\Gamma$ contains more than one subgroup of index 2, then the set of orientifold representations of $\Gamma$ depends on which of these groups is chosen as $\Gamma^{+}$. These facts motivate the definition of an orientifold group.

Definition 1. An orientifold group $(\Gamma, \epsilon)$ is a Lie group equipped with a non-trivial homomorphism $\epsilon: \Gamma \rightarrow \mathbb{Z}_{2}$. For any orientifold group define $\Gamma^{+}:=\operatorname{ker}(\epsilon)$ and $\Gamma^{-}:=\Gamma \backslash \operatorname{ker}(\epsilon)$.

Definition 2. A homomorphism $\varphi:\left(\Gamma^{\prime}, \epsilon^{\prime}\right) \rightarrow(\Gamma, \epsilon)$ of orientifold groups is a group homomorphism such that $\epsilon \circ \varphi=\epsilon^{\prime}$.

The next lemma collects some basic facts about orientifold groups.

Lemma 3. If $(\Gamma, \epsilon)$ is an orientifold group, then

(a) $\Gamma^{+} \subset \Gamma$ is a normal subgroup

(b) $\Gamma / \Gamma^{+} \simeq \mathbb{Z}_{2}$

(c) $1 \rightarrow \Gamma^{+} \rightarrow \Gamma \stackrel{\epsilon}{\rightarrow} \mathbb{Z}_{2} \rightarrow 1$ is an extension of topological groups

(d) $\gamma^{2} \in \Gamma^{+}$for all $\gamma \in \Gamma$

(e) $\Gamma=\Gamma^{+} \sqcup \Gamma^{-}=\Gamma^{+} \sqcup \zeta \Gamma^{+}$for any $\zeta \in \Gamma^{-}$.

The simplest non-trivial example of an orientifold group is provided by id : $\mathbb{Z}_{2} \rightarrow \mathbb{Z}_{2}$. Given an orientifold group, its semi-direct product with a $\Gamma$-group can yield another orientifold group. 
Lemma 4. Let $\epsilon: \Gamma \rightarrow \mathbb{Z}_{2}$ be an orientifold group and $(G, \theta)$ be a $\Gamma$-group. Then the group extension

$$
\begin{gathered}
1 \longrightarrow \Gamma^{+} \ltimes_{\theta} G \stackrel{i}{\longrightarrow} \Gamma \ltimes_{\theta} G \stackrel{\epsilon \circ \pi_{1}}{\longmapsto} \mathbb{Z}_{2} \longrightarrow 1 \\
(\gamma, g) \longmapsto(\gamma, g) \longmapsto \epsilon(\gamma)
\end{gathered}
$$

makes $\Gamma \ltimes_{\theta} G$ into an orientifold group. The notation $(\Gamma, \epsilon) \ltimes_{\theta} G$ will be used to denote orientifold groups of this form.

The following example commonly arises when $G$ is a group of linear operators and $\kappa$ represents conjugation with respect to a fixed basis.

Example 5. Let $(G, \theta)$ be a $\mathbb{Z}_{2}$-group with unit $e$, then $\left(\mathbb{Z}_{2}\right.$, id $) \ltimes_{\theta} G$ is an orientifold group

$$
\begin{gathered}
1 \longrightarrow G \stackrel{i}{\longrightarrow} \mathbb{Z}_{2} \ltimes_{\theta} G \stackrel{\text { ido } \pi_{1}}{\longrightarrow} \mathbb{Z}_{2} \longrightarrow 1 \\
g \longmapsto(z, g) \longmapsto z .
\end{gathered}
$$

Note that the element $(-1, e) \in \Gamma^{-}$is an involution,

$$
(-1, e)^{2}=\left((-1)^{2}, e(-1 e)\right)=\left(-1^{2}, e^{2}\right)=(+1, e) .
$$

It is also possible to construct examples in which $\Gamma^{-}$does not contain an involution.

Example 6. The Weil group $[1, \S X V]$ of $\mathbb{R}$ is the subgroup $\mathbb{C}^{\times} \sqcup \mathbb{C}^{\times} j \subset \mathbb{H}^{\times}$ of the multiplicative group of quaternions. It fits into the non-split extension

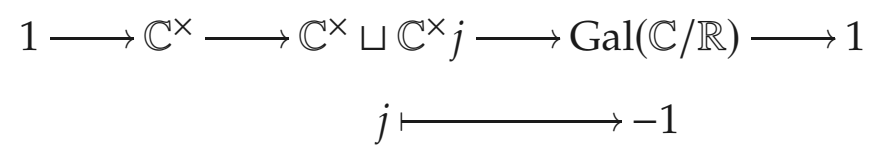

of $\mathbb{C}^{\times}$by $\operatorname{Gal}(\mathbb{C} / \mathbb{R}) \simeq \mathbb{Z}_{2}$, making it into an orientifold group. Note that there is no element $\zeta \in \mathbb{C}^{\times} j=\Gamma^{-}$such that $\zeta^{2}=1$.

Example 7. If $H:=\{ \pm 1, \pm i\}$ is the orientifold group equipped with the homomorphism $q(h):=h^{2}$, then $\Gamma:=(H, q) \ltimes_{\theta} G$ is the orientifold group

$$
\begin{gathered}
1 \longrightarrow\{ \pm 1\} \ltimes_{\theta} G \stackrel{i}{\longrightarrow}\{ \pm 1, \pm i\} \ltimes_{\theta} G \stackrel{q \circ \pi_{1}}{\longrightarrow} \mathbb{Z}_{2} \longrightarrow 1 \\
(h, g) \longmapsto(h, g) \longmapsto h^{2} .
\end{gathered}
$$

If $(h, g) \in \Gamma^{-}$, then $h= \pm i$ and $(h, g)^{2}=\left(h^{2}, g(h g)\right)=(-1, g(h g)) \in \Gamma^{+}$. Thus, there is no element $\gamma \in \Gamma^{-}$such that $\gamma^{2}=(1, e)$. 
Given an orientifold group $(\Gamma, \epsilon)$, the parity information provided by $\epsilon$ can be used when defining actions on various objects. Three different types of actions of an orientifold group will be distinguished. The first type of action uses the parity information assigned to group elements to dictate whether an element acts linearly or anti-linearly. It will be neccesary to define these actions on a variety of $\mathbb{C}$-modules from different categories, including complex vector spaces, complex vector bundles, and algebras over $\mathbb{C}$. Given objects $X$ and $Y$ in an appropriate category, define

$$
\begin{aligned}
& \operatorname{Hom}^{+1}(X, Y):=\operatorname{Hom}(X, Y) \\
& \operatorname{Hom}^{-1}(X, Y):=\left\{a_{\bar{Y}} \circ \varphi \mid \varphi \in \operatorname{Hom}(X, \bar{Y})\right\}
\end{aligned}
$$

where $a_{\bar{Y}}: \bar{Y} \rightarrow Y$ is the identity map on the underlying set for $Y$. The map $a_{\bar{Y}}$ is anti-linear and the elements of $\operatorname{Hom}^{-1}(X, Y)$ can be considered as anti-linear homomorphisms. The conjugation map $Y \mapsto \bar{Y}$ changes the $\mathbb{C}$-module structure of $Y$ to its conjugate $\mathbb{C}$-module structure, and, depending on the category, it may change other structures on $Y$. For example, the conjugate of a Hilbert space carries a conjugate inner product. Denote the disjoint union of $\mathrm{Hom}^{+}$and $\mathrm{Hom}^{-}$by Hom $^{ \pm}$. The spaces End ${ }^{ \pm}$and Aut $^{ \pm}$are defined similarly.

Definition 8. Let $(\Gamma, \epsilon)$ be an orientifold group. An orientifold action is a homomorphism $\rho: \Gamma \rightarrow \operatorname{Aut}^{ \pm}(W)$ such that

$$
\rho(\gamma) \in \operatorname{Aut}^{\epsilon(\gamma)}(W)
$$

A second type of action uses an involution $\rho$ to define an action of $\Gamma$. Typically, an involution of this type represents the change of some structure to a conjugate structure, occuring in parallel with the application of an orientifold action.

Definition 9. An involutive action of an orientifold group, is an action of the form

$$
\rho \circ \epsilon: \Gamma \rightarrow \mathbb{Z}_{2} \rightarrow \operatorname{Aut}(Y),
$$

where $\rho: \mathbb{Z}_{2} \rightarrow \operatorname{Aut}(Y)$ is an involution.

Example 10. Some examples of involutive actions are

(a) $\iota_{\epsilon}^{p, q}: \mathbb{R}^{p, q} \rightarrow \mathbb{R}^{p, q}$, where $\mathbb{R}^{p, q}:=\mathbb{R}^{p} \oplus \mathbb{R}^{q}, \iota^{p, q}:(x, y) \mapsto(x,-y)$.

(b) $\kappa_{\epsilon}: \operatorname{GL}(n, \mathbb{C}) \rightarrow \mathrm{GL}(n, \mathbb{C})$, where $\kappa_{\epsilon}$ is elementwise conjugation on the standard matrix representation of $\operatorname{GL}(n, \mathbb{C})$. 
(c) $d \theta_{\epsilon}: \mathfrak{g} \rightarrow \mathfrak{g}$, where $\mathfrak{g}$ is a Lie algebra and $\theta: G \rightarrow G$ is an involution on its Lie group.

Throughout this paper, it will be assumed that $\mathbb{C}$ is equipped with the orientifold action $\kappa_{\epsilon}$.

Of course, the parity of the group elements can also be ignored. This type of action occurs on an orientifold and its tangent bundle. In order to differentiate it from the other types of action, it will be refered to as a basic action.

2.2. Orientifolds. In order to maintain a clear focus on anti-linear symmetry, only the simplest definition of an orientifold will be treated. These orientifolds are essentially global quotient orbifolds with a small amount of extra structure. Using the language of $\$ 2.1$, they could be described as manifolds equipped with a basic action of an orientifold group. The origin of the term orientifold is in string theory, where orientifolds are often considered to have a sign choices \pm 1 associated to the connected components of their fixed point sets. However, these sign choice structures will not be considered here.

Definition 11. An orientifold is a compact manifold $X$ equipped with a smooth action

$$
\rho: \Gamma \rightarrow \operatorname{Diff}(X),
$$

where $\Gamma$ is a finite orientifold group. The category of orientifolds with orientifold group $\epsilon: \Gamma \rightarrow \mathbb{Z}_{2}$ will be denoted $\operatorname{Ori}_{(\Gamma, \epsilon)}$.

Example 12. Let $\Gamma$ be any orientifold group. Then $\mathbb{R}^{p, q}:=\mathbb{R}^{p} \oplus \mathbb{R}^{q}$ equipped with the involutive action induced by $(x, y) \mapsto(x,-y)$ is an orientifold. This orientifold will be used to form suspensions in orientifold K-theory.

Example 13. Let $X \in \operatorname{Ori}_{(\Gamma, \epsilon)}$ with $\Gamma$-action $\sigma$. The tangent bundle $T X$ equipped with the basic $\Gamma$-action $d \sigma$ is again an orientifold. The $K$-theory of this orientifold is the target space of the 8-fold Thom isomorphism in orientifold $K$-theory.

The category of real vector bundles equipped with a basic action of the orientifold group $(\Gamma, \epsilon)$ will be denoted $\operatorname{Vect}_{(\Gamma, \epsilon)}(X, \mathbb{R})$. The isomorphism classes of such bundles will be denoted $\operatorname{Vect}_{(\Gamma, \epsilon)}^{\simeq}(X, \mathbb{R})$.

2.3. Orientifold Bundles. Orientifold bundles are the main object of interest in the study of orientifolds. In the language of $\$ 2.1$, they are complex vector bundles carrying orientifold actions that cover the action on the base orientifold. 
Definition 14. If $\pi: E \rightarrow X$ is a complex vector bundle, define $\operatorname{Aut}_{\text {Diff }}(E)$ to be the set of maps $\varphi: E \rightarrow E$ such that

(a) $\pi \circ \varphi(e)=f \circ \pi(e)$, for some $f \in \operatorname{Diff}(X)$ and all $e \in E$.

(b) $\varphi: E_{x} \rightarrow E_{f(x)}$ is a linear bijection, for all $x$.

Definition 15. An orientifold bundle $\pi: E \rightarrow X$ is a complex vector bundle equipped with an orientifold action

$$
\tau: \Gamma \rightarrow \operatorname{Aut}_{\mathrm{Diff}}^{ \pm}(E)
$$

such that $\pi(\gamma v)=\gamma \pi(v)$.

The category of orientifold bundles over $X \in \operatorname{Ori}_{(\Gamma, \epsilon)}$ will be denoted $\operatorname{Vect}_{(\Gamma, \epsilon)}(X, \mathbb{C})$. The set of isomorphism classes of orientifold bundles will be denoted $\operatorname{Vect}_{(\Gamma, \epsilon)}^{\simeq}(X, \mathbb{C})$.

Example 16. A linear/anti-linear representation $(V, \rho)$ is an orientifold bundle over a point. Such a bundle will also be refered to as an orientifold representation. If $(X, \sigma)$ is an orientifold and $(V, \rho)$ is an orientifold representation, then an orientifold bundle of the form

$$
(X \times V, \sigma \times \rho)
$$

will be described as a trivial orientifold bundle. Note that if $\epsilon$ is nontrivial, then every orientifold bundle for $(\Gamma, \epsilon)$ carries at least one antilinear map, and so there is no orientifold bundle $(E, \tau)$ such that $\tau_{\gamma}=\mathrm{id}$ for all $\gamma \in \Gamma$.

Just as in the equivariant setting, it is possible to average an hermetian metric on an orientifold bundle to make it compatible with the orientifold action. The averaging process needs to be twisted with conjugation to account for the anti-linearity of the action, as does the compatibility condition.

Definition 17. An orientifold metric on an orientifold bundle $E$ is an hermitian metric $h$ on $E$ such that, for all $v_{1}, v_{2} \in E$ and $\gamma \in \Gamma$,

$$
h\left(\gamma v_{1}, \gamma v_{2}\right)_{\gamma x}=\gamma h\left(v_{1}, v_{2}\right)_{x}
$$

Proposition 18. Every orientifold vector bundle E over a paracompact orientifold $X$ carries an orientifold metric. 
Proof. It is a standard result that every complex vector bundle over a paracompact space carries an hermitian metric [27, Lemma 2]. Given an hermitian metric $h$ on an orientifold bundle $E$, define

$$
h_{\Gamma}(u, v)_{x}=\sum_{\gamma \in \Gamma} \gamma^{-1} h(\gamma u, \gamma v)_{\gamma x} .
$$

This metric is an orientifold metric as

$$
\begin{aligned}
h_{\Gamma}(\gamma u, \gamma v)_{\gamma x}=\sum_{\gamma^{\prime} \in \Gamma} \gamma^{\prime-1} h\left(\gamma^{\prime} \gamma u, \gamma^{\prime} \gamma v\right)_{\gamma^{\prime} \gamma x} & \\
= & \sum_{\gamma^{\prime \prime}:=\gamma^{\prime} \gamma \in \Gamma} \gamma \gamma^{\prime \prime-1} h\left(\gamma^{\prime \prime} u, \gamma^{\prime \prime} v\right)_{\gamma^{\prime \prime} x} \\
& =\gamma \sum_{\gamma^{\prime \prime} \in \Gamma} \gamma^{\prime \prime-1} h\left(\gamma^{\prime \prime} u, \gamma^{\prime \prime} v\right)_{\gamma^{\prime \prime} x}=\gamma h_{\Gamma}(u, v)_{x} .
\end{aligned}
$$

Using an orientifold metric, it is possible to split sequences of orientifold bundles.

Corollary 19. Let $X$ be a paracompact orientifold. If

$$
0 \rightarrow E^{\prime} \stackrel{\varphi^{\prime}}{\rightarrow} E \stackrel{\varphi}{\rightarrow} E^{\prime \prime}
$$

is an exact sequence of orientifold bundles over $X$, then $E \simeq E^{\prime} \oplus E^{\prime \prime}$.

Proof. By Proposition 18, there exists an orientifold metric $h$ on $E$. It is a standard result [27, Proposition 2] that $h$ determines a projection $p: E \rightarrow E$ and a splitting of complex vector bundles $E=\operatorname{im}(p) \oplus$ $\operatorname{ker}(p) \simeq E^{\prime} \oplus E^{\prime \prime}$. The projection $p$ is defined fibrewise by

$$
\begin{aligned}
p_{x}: E_{x} & \rightarrow E_{x} \\
v & \mapsto \sum_{i} \frac{h\left(v, b_{i}\right)_{x}}{h\left(b_{i}, b_{i}\right)_{x}} b_{i},
\end{aligned}
$$

where $\left\{b_{i}\right\}$ is any basis for $\varphi^{\prime}\left(E^{\prime}\right)_{x}$. Therefore, if $p_{x}(v)=0$, then $h\left(v, b_{i}\right)_{x}=0$ for all $i$, and

$$
\begin{aligned}
p_{\gamma x}(\gamma v)=\sum_{i} \frac{h\left(\gamma v, \gamma b_{i}\right)_{\gamma x}}{h\left(\gamma b_{i}, \gamma b_{i}\right)_{\gamma x}}\left(\gamma b_{i}\right) \\
=\sum_{i} \frac{\gamma h\left(v, b_{i}\right)_{x}}{\gamma h\left(b_{i}, b_{i}\right)_{x}}\left(\gamma b_{i}\right)=\sum_{i} \frac{\gamma 0}{\gamma h\left(b_{i}, b_{i}\right)_{x}}\left(\gamma b_{i}\right)=0 .
\end{aligned}
$$

Thus, $\operatorname{ker}(p)$ is invariant under the action of $\Gamma$, as is the given splitting. 
Next, the frame bundle of an orientifold bundle will be examined.

Definition 20. The frame bundle $\operatorname{Fr}(E)$ of an orientifold bundle $E$ is the principal $\operatorname{GL}(n, \mathbb{C})$-bundle of frames for the total space of $E$, equipped with a left $\Gamma$-action defined on a frame $s=\left(s_{1}, \ldots, s_{n}\right) \in \operatorname{Fr}(E)_{x}$ by $(\gamma s)_{i}=\gamma s_{i}$.

Although the frame bundle of an orientifold is defined in the same manner as that of an equivariant bundle, the anti-linearity present in the $\Gamma$-action gives it different properties. In particular, there is a mild noncommutivity between the left action of $\Gamma$ and the right action of the structure group $\mathrm{GL}(n, \mathbb{C})$. This non-commutivity makes the frame bundle of an orientifold bundle into a $\Gamma$-semi-equivariant principal $\left(\mathrm{GL}(n, \mathbb{C}), \kappa_{\epsilon}\right)$-bundle [21, §2].

Proposition 21. Let $E$ be an orientifold bundle and consider $\mathrm{GL}(n, \mathbb{C})$ to be equipped with the involutive action of $(\Gamma, \epsilon)$ induced by conjugation. Then,

$$
\operatorname{Fr}(E ; \operatorname{GL}(n, \mathbb{C})) \in \operatorname{PB}_{\Gamma}\left(X,\left(\operatorname{GL}(n, \mathbb{C}), \kappa_{\epsilon}\right)\right) .
$$

In particular, the left and right actions on the frame bundle satisfy

$$
\gamma(s g)=(\gamma s)(\gamma g)
$$

for $\gamma \in \Gamma, s \in \operatorname{Fr}(E)$ and $g \in \operatorname{GL}(n, \mathbb{C})$.

Proof. The action of $g$ on a frame $s$ is given by $(s g)_{j}=\sum_{1 \leq i \leq n} s_{i} g_{i j}$. Thus,

$$
\begin{gathered}
\gamma(s g)_{j}=\sum_{1 \leq i \leq n} \gamma\left(s_{i} g_{i j}\right)=\sum_{1 \leq i \leq n}\left(\gamma s_{i}\right)\left(\gamma g_{i j}\right)=\sum_{1 \leq i \leq n}(\gamma s)_{i}(\gamma g)_{i j} \\
=((\gamma s)(\gamma g))_{j} .
\end{gathered}
$$

Note that, by using an orientifold metric, the structure group can always be reduced to $\left(\mathrm{U}(n), \kappa_{\epsilon}\right)$, where $\kappa_{\epsilon}$ is the action induced on $\mathrm{U}(n)$ by its inclusion into $\mathrm{GL}(n, \mathbb{C})$.

2.4. Operations on Orientifold Bundles. Some basic operations on orientifold bundles will now be defined. It will be useful to make these definitions in terms of semi-equivariant cocycles [21, §3]. To start with, consider the following operations on $\Gamma$-groups.

Definition 22. Let $a^{k} \in \mathrm{GL}\left(\mathbb{C}^{m_{k}}\right)$, and denote by $\left[a_{i j}\right]$ the matrix representation of an element $a \in \mathrm{GL}\left(\mathbb{C}^{m}\right)$ with respect to the standard basis of $\mathbb{C}^{m}$. Define the following operations 
(a) The dual $a^{*} \in \mathrm{GL}\left(\mathbb{C}^{m}\right)$,

$$
\left[\left(a^{*}\right)_{i j}\right]:=\left(\left[a_{i j}\right]^{t}\right)^{-1}
$$

(b) The direct sum $a^{1} \oplus a^{2} \in \mathrm{GL}\left(\mathbb{C}^{m_{1}+m_{2}}\right)$,

$$
\left[\left(a^{1} \oplus a^{2}\right)_{i j}\right]:=\left(\begin{array}{cc}
{\left[a_{i j}^{1}\right]} & 0 \\
0 & {\left[a_{i j}^{2}\right]}
\end{array}\right) .
$$

(c) The tensor product $a^{1} \otimes a^{2} \in \mathrm{GL}\left(\mathbb{C}^{m_{1} m_{2}}\right)$,

$$
\left[\left(a^{1} \otimes a^{2}\right)_{i j}\right]:=\left(\begin{array}{ccc}
a_{11}^{1}\left[a_{i j}^{2}\right] & \ldots & a_{1 m}^{1}\left[a_{i j}^{2}\right] \\
\vdots & \ddots & \vdots \\
a_{m 1}^{1}\left[a_{i j}^{2}\right] & \ldots & a_{m m}^{1}\left[a_{i j}^{2}\right]
\end{array}\right) .
$$

Examining Definition 22, it is clear that the dual, direct sum and tensor product on the groups $\mathrm{GL}\left(\mathbb{C}^{m}\right)$ are compatible with involutive $\Gamma$-actions induced by conjugation.

Lemma 23. The dual, direct sum and tensor product operations are homomorphisms

$$
\begin{aligned}
& *:\left(\mathrm{GL}\left(\mathbb{C}^{m}\right), \kappa_{\epsilon}\right) \rightarrow\left(\mathrm{GL}\left(\mathbb{C}^{m}\right), \kappa_{\epsilon}\right) \\
& \oplus:\left(\mathrm{GL}\left(\mathbb{C}^{m_{1}}\right), \kappa_{\epsilon}\right) \times\left(\mathrm{GL}\left(\mathbb{C}^{m_{2}}\right), \kappa_{\epsilon}\right) \rightarrow\left(\mathrm{GL}\left(\mathbb{C}^{m_{1}+m_{2}}\right), \kappa_{\epsilon}\right) \\
& \otimes:\left(\mathrm{GL}\left(\mathbb{C}^{m_{1}}\right), \kappa_{\epsilon}\right) \times\left(\mathrm{GL}\left(\mathbb{C}^{m_{2}}\right), \kappa_{\epsilon}\right) \rightarrow\left(\mathrm{GL}\left(\mathbb{C}^{m_{1} m_{2}}\right), \kappa_{\epsilon}\right)
\end{aligned}
$$

of $\Gamma$-groups.

Lemma 23, allows the dual, direct sum and tensor product of $\left(\mathrm{GL}(m, \mathbb{C}), \kappa_{\epsilon}\right)$-valued transition cocycles [21, §3] to be defined in the obvious way. Pullbacks of cocycles can also be defined. It is routine to prove that these satisfy the semi-equivariant cocycle condition.

Definition 24. Let $\phi^{i} \in \mathrm{TC}_{(\Gamma, \epsilon)}\left(\mathcal{U}, X,\left(\mathrm{GL}\left(\mathbb{C}^{m_{i}}\right), \kappa_{\epsilon}\right)\right)$ and $f: X \rightarrow Y$ be a homomorphism orientifolds. The pullback, dual, direct sum, and tensor product are defined, respectively, by

$$
\begin{aligned}
& \left(f^{*} \phi\right)_{b a}(\gamma, x):=\phi_{b a}(\gamma, f(x)) \quad \in \operatorname{TC}_{(\Gamma, \epsilon)}\left(f^{*} \mathcal{U}, Y_{,}\left(\mathrm{GL}\left(\mathbb{C}^{m}\right), \kappa_{\epsilon}\right)\right) \\
& \left(\phi^{*}\right)_{b a}(x, \gamma):=\phi_{b a}(x, \gamma)^{*} \quad \in \operatorname{TC}_{(\Gamma, \epsilon)}\left(\mathcal{U}, X,\left(\operatorname{GL}\left(\mathbb{C}^{m}\right), \kappa_{\epsilon}\right)\right) \\
& \left(\phi^{1} \oplus \phi^{2}\right)_{b a}(x, \gamma):=\phi_{b a}^{1}(x, \gamma) \oplus \phi_{b a}^{2}(x, \gamma) \in \operatorname{TC}_{(\Gamma, \epsilon)}\left(\mathcal{U}, X,\left(\operatorname{GL}\left(\mathbb{C}^{m_{1}+m_{2}}\right), \kappa_{\epsilon}\right)\right) \\
& \left(\phi^{1} \otimes \phi^{2}\right)_{b a}(x, \gamma):=\phi_{b a}^{1}(x, \gamma) \otimes \phi_{b a}^{2}(x, \gamma) \in \operatorname{TC}_{(\Gamma, \epsilon)}\left(\mathcal{U}, X,\left(\mathrm{GL}\left(\mathbb{C}^{m_{1} m_{2}}\right), \kappa_{\epsilon}\right)\right), \\
& \text { where } f^{*} \mathcal{U}:=\left\{f^{-1}\left(U_{a}\right) \mid a \in A\right\} \text { is the pullback of } \mathcal{U}:=\left\{U_{a} \mid a \in A\right\} \text {. }
\end{aligned}
$$


The above operations on cocycles induce operations on orientifold bundles via the semi-equivariant associated bundle construction, see Definition 59.

Definition 25. Let $E_{i} \in \operatorname{Vect}_{(\Gamma, \epsilon)}^{m_{i}}(X, \mathbb{C})$. Let $\phi^{i}$ denote a semi-equivariant cocycle associated $\operatorname{Fr}\left(E_{i}\right)$ by [21, Prop. 12], and $P^{\phi}$ denote the semiequivariant principal bundle constructed from a cocycle $\phi$ via [21, Prop. 15]. $P^{\phi}$ denote The pullback, dual, direct sum, and tensor product operations on orientifold bundles are defined, respectively, by

$$
\begin{array}{rlrl}
f^{*} E & :=P^{f^{*} \phi} \times_{\left(\mathrm{GL}(m, \mathbb{C}), \kappa_{\epsilon}\right)}\left(\mathbb{C}^{m}, \kappa_{\epsilon}\right) & \in \operatorname{Vect}_{(\Gamma, \epsilon)}^{m}(X, \mathbb{C}) \\
E^{*}:=P^{\phi^{*}} \times_{\left(\mathrm{GL}(m, \mathbb{C}), \kappa_{\epsilon}\right)}\left(\left(\mathbb{C}^{m}\right)^{*}, \kappa_{\epsilon}\right) & \in \operatorname{Vect}_{(\Gamma, \epsilon)}^{m}(X, \mathbb{C}) \\
E_{1} \oplus E_{2}:=P^{\phi_{1} \oplus \phi_{2}} \times_{\left(\mathrm{GL}\left(m_{1}+m_{2}, \mathbb{C}\right), \kappa_{\epsilon}\right)}\left(\mathbb{C}^{m_{1}+m_{2}}, \kappa_{\epsilon}\right) & \in \operatorname{Vect}_{(\Gamma, \epsilon)}^{m_{1}+m_{2}}(X, \mathbb{C}) \\
E_{1} \otimes E_{2}: & :=P^{\phi_{1} \otimes \phi_{2}} \times_{\left(\mathrm{GL}\left(m_{1} m_{2}, \mathbb{C}\right), \kappa_{\epsilon}\right)}\left(\mathbb{C}^{m_{1} m_{2}}, \kappa_{\epsilon}\right) & \in \operatorname{Vect}_{(\Gamma, \epsilon)}^{m_{1} m_{2}}(X, \mathbb{C}),
\end{array}
$$

where $\kappa_{\epsilon}:\left(\mathbb{C}^{m}\right)^{*} \rightarrow\left(\mathbb{C}^{m}\right)^{*}$ is the action defined by $(\gamma \lambda)(z):=\gamma \lambda\left(\gamma^{-1} z\right)$.

As in the non-equivariant setting, it is possible to construct the bundle of homomorphisms between two orientifold bundles using their tensor products and duals.

Proposition 26. Let $E_{i} \in \operatorname{Vect}_{(\Gamma, \epsilon)}^{m_{i}}(X, \mathbb{C})$. Homomorphisms in $\operatorname{Hom}\left(E_{1}, E_{2}\right)$ correspond bijectively to equivariant sections of the orientifold bundle $E_{2} \otimes E_{1}^{*}$.

2.5. Classification of Spin ${ }^{\mathrm{c}}$-Structures on Orientifolds. In order to define and classify Spin ${ }^{\mathrm{C}}$-structures for orientifolds, it is neccesary to consider the interaction of Clifford algebras and the Spin groups with orientifold actions. The idea is to complexify results which apply to real Clifford algebras, whilst keeping track of the associated conjugation maps. These maps can then be used to define involutive actions of orientifold groups. To begin, the definitions of the real Clifford algebra, Spin group, and adjoint map are recalled.

Definition 27. The Clifford algebra $\mathrm{Cl}_{n}$ is the algebra generated by the standard basis $\left\{e_{i}\right\}$ of $\mathbb{R}^{n}$ subject to the relations $e_{i}^{2}=-1$ and $e_{i} e_{j}+e_{j} e_{i}=0$.

Note that the set $\left\{e_{i_{1}} \cdots e_{i_{k}} \in \mathrm{Cl}_{n} \mid i_{1}<\cdots<i_{k}\right\}$ is a basis for $\mathrm{Cl}_{n}$. The group $\operatorname{Spin}(n)$ sits inside $\mathrm{Cl}_{n}$. Elements of $\operatorname{Spin}(n)$ are products of an even number of unit vectors from $\mathbb{R}^{n}$.

Definition 28. The group $\operatorname{Spin}(n)$ is defined by

$$
\operatorname{Spin}(n):=\left\{x_{1} \cdots x_{2 k} \mid x_{i} \in \mathbb{R}^{n},\left\|x_{i}\right\|=1\right\} \subset \mathrm{Cl}_{n} .
$$


If $g \in \operatorname{Spin}(n)$ and $x \in \mathbb{R}^{n}$ one can show that $g x g^{-1} \in \mathbb{R}^{n}$. The transformation $x \mapsto g x g^{-1}$ defines an element of $\mathrm{SO}(n)$, and the resulting assignment $\operatorname{Spin}(n) \rightarrow \mathrm{SO}(n)$ is a double covering.

Definition 29. The adjoint map Ad $: \operatorname{Spin}(n) \rightarrow \mathrm{SO}(n)$ is defined, for $g \in \operatorname{Spin}(n), x \in \mathbb{R}^{n}$, by

$$
\operatorname{Ad}_{g}(x):=g x g^{-1}
$$

For applications to orientifolds, it is neccesary to work with the complexifications of $\mathrm{Cl}_{n}$ and $\operatorname{Spin}(n)$. These complexifications are equipped with conjugation maps which induce involutive actions of orientifold groups. The complexified adjoint map is a homomorphism of $\Gamma$-groups.

Definition 30. Let $(\Gamma, \epsilon)$ be an orientifold group and define the following

(a) $\left(\mathbb{C l}_{n}, \kappa_{\epsilon}\right):=\mathrm{Cl}_{n} \otimes \mathbb{C}$ with the $\Gamma$-action $\kappa_{\epsilon}(\varphi \otimes z):=\varphi \otimes \kappa_{\epsilon}(z)$

(b) $\left(\operatorname{Spin}^{\mathrm{c}}(n), \kappa_{\epsilon}\right):=(\operatorname{Spin}(n) \times \mathrm{U}(1)) /\{ \pm(1,1)\}$ with the induced action $\kappa_{\epsilon}[g, z]:=\left[g, \kappa_{\epsilon}(z)\right]$

(c) $\operatorname{Ad}^{c}:\left(\operatorname{Spin}^{c}(n), \kappa_{\epsilon}\right) \rightarrow\left(\operatorname{SO}(n), \operatorname{id}_{\epsilon}\right)$ defined by $\operatorname{Ad}^{c}[g, z]:=\operatorname{Ad}(g)$.

Note that $\operatorname{Ad}^{c} \circ \kappa_{\epsilon}[g, z]=\operatorname{Ad}^{c}[g, z]$. The properties of $\operatorname{Ad}^{c}$, and the decomposition of $\operatorname{Spin}^{c}(n)$, produce two central exact sequences of $\Gamma$-groups about $\operatorname{Spin}^{c}(n)$. These sequences fit into the following diagram

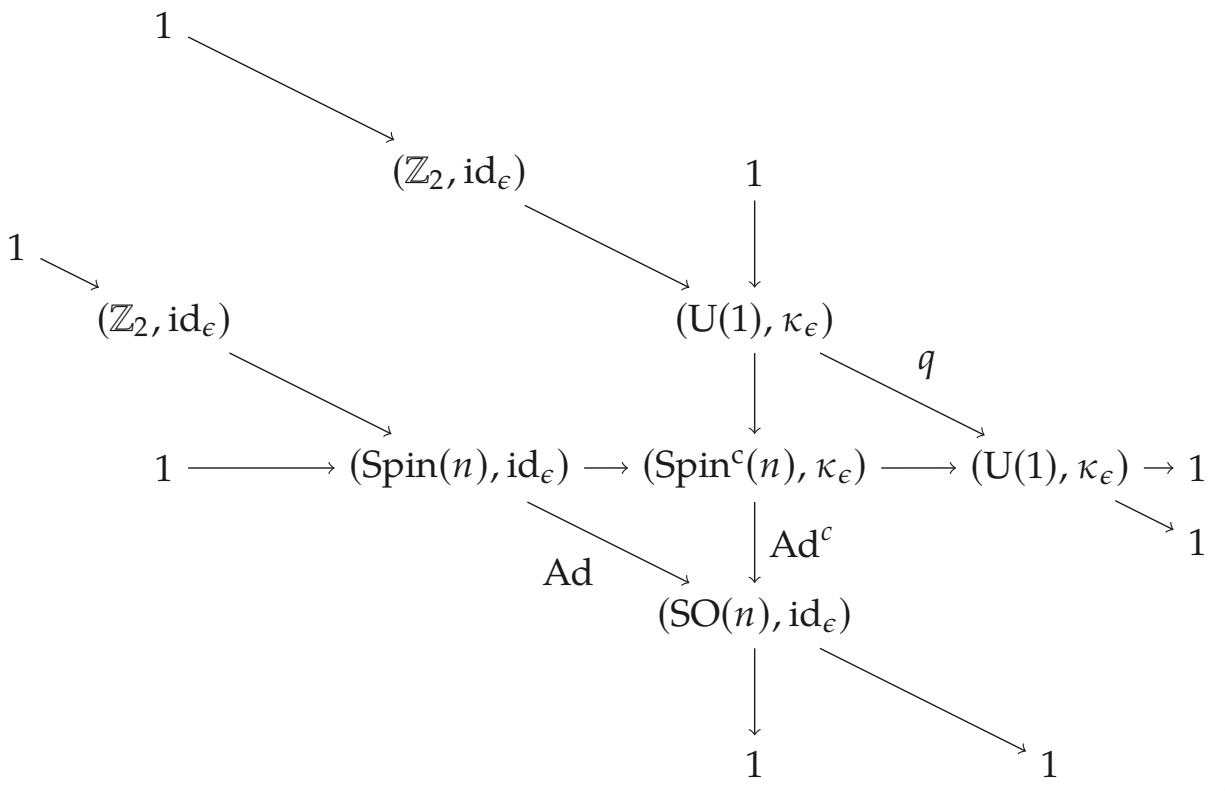


where $q$ is the square map. The above sequences will be used to classify Spin ${ }^{\mathrm{C}}$-structures for orientifolds.

Having examined semi-equivariance, orientifolds, and orientifold actions on $\operatorname{Spin}^{c}(n)$, it is now possible to define a notion of Spincstructure which is appropriate for orientifolds.

Definition 31. An $\left(\operatorname{Spin}^{\mathrm{c}}, \kappa_{\epsilon}\right)$-structure for a real $\Gamma$-equivariant vector bundle $V$ over an orientifold is a semi-equivariant lifting $\varphi: P \rightarrow \operatorname{Fr}(V)$ by $\operatorname{Ad}^{c}:\left(\operatorname{Spin}^{\mathrm{c}}(n), \kappa_{\epsilon}\right) \rightarrow\left(\mathrm{SO}(n), \mathrm{id}_{\epsilon}\right)$.

If $V$ has a $\left(\operatorname{Spin}^{\mathrm{c}}, \kappa_{\epsilon}\right)$-structure, then it is said to be $\left(\operatorname{Spin}^{\mathrm{C}}, \kappa_{\epsilon}\right)$ oriented. If the tangent bundle $T M$ of an orientifold $M$ is $\left(\operatorname{Spin}^{\mathrm{C}}, \kappa_{\epsilon}\right)$ oriented, then $M$ is said to be $\left(\operatorname{Spin}^{\mathrm{c}}, \kappa_{\epsilon}\right)$-oriented.

The $\left(\operatorname{Spin}^{\mathrm{c}}, \kappa_{\epsilon}\right)$-structures associated to a vector bundle $V$ can be classified using the results of [21]. The following theorem is obtained by applying [21, Theorem 41] to the central exact sequence running vertically in diagram (2).

Theorem 32. The central exact sequence

$$
1 \rightarrow\left(\mathrm{U}(1), \kappa_{\epsilon}\right) \rightarrow\left(\operatorname{Spin}^{c}(n), \kappa_{\epsilon}\right) \stackrel{\mathrm{Ad}^{c}}{\rightarrow}\left(\mathrm{SO}(n), \mathrm{id}_{\epsilon}\right) \rightarrow 1,
$$

induces an exact sequence

$$
\begin{aligned}
& H_{\Gamma}^{1}\left(X,\left(\mathrm{U}(1), \kappa_{\epsilon}\right)\right) \rightarrow \mathrm{TC}_{\Gamma}^{1}\left(X,\left(\operatorname{Spin}^{\mathrm{c}}(n), \kappa_{\epsilon}\right)\right) \stackrel{\mathrm{Ad}^{\mathrm{c}}}{\rightarrow} \ldots \\
& \ldots \stackrel{\mathrm{Ad}^{c}}{\rightarrow} \mathrm{TC}_{\Gamma}^{1}\left(X,\left(\mathrm{SO}(n), \mathrm{id}_{\epsilon}\right)\right) \stackrel{\Delta_{s c}}{\rightarrow} H_{\Gamma}^{2}\left(X,\left(\mathrm{U}(1), \kappa_{\epsilon}\right)\right) .
\end{aligned}
$$

Theorem 32 has the following corollaries, which classify $\left(\operatorname{Spin}^{\mathrm{c}}, \kappa_{\epsilon}\right)$ structures in terms of semi-equivariant cohomology with coefficients in $\left(\mathrm{U}(1), \kappa_{\epsilon}\right)$.

Corollary 33. A real $\Gamma$-equivariant vector bundle $V$ over an orientifold has a $\left(\operatorname{Spin}^{\mathrm{c}}, \kappa_{\epsilon}\right)$-structure if and only if $\Delta_{s c}\left(\phi^{V}\right)=1$, where $\phi^{V}$ is the transition cocycle for $V$.

Corollary 34. A given (Spin $\left.{ }^{\mathrm{C}}, \kappa_{\epsilon}\right)$-structure is unique up to tensoring by semi-equivariant principal $\left(\mathrm{U}(1), \kappa_{\epsilon}\right)$-bundles.

To obtain an obstruction class with integer coefficients, involutive actions can be taken on the groups in the exponential exact sequence. This results in the following proposition.

Lemma 35. The exponential exact sequence

$$
0 \rightarrow\left(\mathbb{Z}, \iota_{\epsilon}\right) \rightarrow\left(\mathbb{R}, \iota_{\epsilon}\right) \stackrel{\exp }{\rightarrow}\left(\mathrm{U}(1), \kappa_{\epsilon}\right) \rightarrow 1
$$


induces isomorphisms

$$
H_{\Gamma}^{p}\left(X,\left(\mathrm{U}(1), \kappa_{\epsilon}\right)\right) \stackrel{\Delta_{\exp }^{p}}{\rightarrow} H_{\Gamma}^{p+1}\left(X,\left(\mathbb{Z}, \iota_{\epsilon}\right)\right),
$$

where $\iota_{\epsilon}$ is the involutive orientifold action induced by the map $t \mapsto-t \in \mathbb{R}$.

Proof. By [21, Theorem 38], the exact sequence (3) induces a long exact sequence

$H_{\Gamma}^{p}\left(X,\left(\mathbb{Z}, \iota_{\epsilon}\right)\right) \rightarrow H_{\Gamma}^{p}\left(X,\left(\mathbb{R}, \iota_{\epsilon}\right)\right) \stackrel{\exp }{\rightarrow} H_{\Gamma}^{p}\left(X,\left(\mathrm{U}(1), \kappa_{\epsilon}\right)\right) \stackrel{\Delta_{\exp }^{p}}{\rightarrow} H_{\Gamma}^{p+1}\left(X,\left(\mathbb{Z}, \iota_{\epsilon}\right)\right)$.

The cohomology groups $H_{\Gamma}^{p}\left(X,\left(\mathbb{R}, \iota_{\epsilon}\right)\right)$ vanish for all $p$, due to the existence of a smooth partition of unity on $X$. Therefore, the maps $\Delta_{\exp }^{p}$ are isomorphisms.

Using Lemma 35, it is possible to define an analogue of the third integral Stiefel-Whiney class.

Definition 36. The third integral orientifold Stiefel-Whiney class is defined by

$$
W_{3}^{(\Gamma, \epsilon)}(V):=\Delta_{\exp } \circ \Delta_{s c}\left(\phi^{V}\right) \in H_{\Gamma}^{3}\left(X,\left(\mathbb{Z}, \iota_{\epsilon}\right)\right),
$$

where $\phi^{V}$ is the transition cocycle associated to $V$.

Corollaries 33 and 34 can then be restated in terms of semi-equivariant cohomology with coefficients in $\left(\mathbb{Z}, \iota_{\epsilon}\right)$.

Corollary 37. A real $\Gamma$-equivariant bundle $V$ is $\left(\operatorname{Spin}^{\mathrm{c}}, \kappa_{\epsilon}\right)$-oriented if and only if $W_{3}^{(\Gamma, \epsilon)}(V)=0$.

Corollary 38. The $\left(\operatorname{Spin}^{\mathrm{c}}, \kappa_{\epsilon}\right)$-structures on a $\left(\operatorname{Spin}^{\mathrm{c}}, \kappa_{\epsilon}\right)$-oriented real $\Gamma$ equivariant vector bundle are in bijective correspondence with the elements of $H_{\Gamma}^{2}\left(X,\left(\mathbb{Z}, \iota_{\epsilon}\right)\right)$.

It is possible to further isolate the semi-equivariance in a $\left(\operatorname{Spin}^{\mathrm{c}}, \kappa_{\epsilon}\right)$ structure by splitting it via the decomposition

$$
\left(\operatorname{Spin}^{\mathrm{c}}(n), \kappa\right) \simeq(\mathrm{SO}(n), \mathrm{id}) \times_{\mathbb{Z}_{2}}(\mathrm{U}(1), \kappa) .
$$

This decomposition immediately implies that, for any cochain $\phi_{s c} \in$ $K_{\Gamma}^{1}\left(X,\left(\operatorname{Spin}^{c}(n), \kappa_{\epsilon}\right)\right)$, there exist cochains $\phi_{s} \in K_{\Gamma}^{1}\left(X,\left(\operatorname{Spin}(n), \operatorname{id}_{\epsilon}\right)\right)$ and $\phi_{u} \in K_{\Gamma}^{1}\left(X,\left(\mathrm{U}(1), \kappa_{\epsilon}\right)\right)$ such that $\phi_{s c}=\left[\phi_{s}, \phi_{u}\right]$. It also allows the definition of the map

$$
\begin{aligned}
\operatorname{Ad} \times q:\left(\operatorname{Spin}^{c}(n), \kappa_{\epsilon}\right) & \rightarrow\left(\operatorname{SO}(n), \operatorname{id}_{\epsilon}\right) \times\left(\mathrm{U}(1), \kappa_{\epsilon}\right) \\
{[s, z] } & \mapsto(\operatorname{Ad}(s), q(z)) .
\end{aligned}
$$

The next proposition shows that every $\left(\operatorname{Spin}^{\mathrm{C}}, \kappa_{\epsilon}\right)$-structure extends to a lifting of a semi-equivariant principal $(\mathrm{SO}(n), \mathrm{id}) \times(\mathrm{U}(1), \kappa)$-bundle by $\operatorname{Ad} \times q$. 
Proposition 39. If $\varphi_{0}: P \rightarrow Q$ is a (Spin ${ }^{\mathrm{c}}, \kappa_{\epsilon}$ )-structure, then there exists a lifting

$$
\varphi: P \rightarrow Q \times_{X} L
$$

by $\mathrm{Ad} \times q$, where $L$ is a $\Gamma$-semi-equivariant principal $\left(\mathrm{U}(1), \kappa_{\epsilon}\right)$-bundle.

Proof. Let $\phi \in \mathrm{TC}_{\Gamma}^{1}\left(X,\left(\mathrm{SO}(n), \mathrm{id}_{\epsilon}\right)\right)$ be the cocycle for $Q$. If $Q$ has a $\left(\operatorname{Spin}^{\mathrm{c}}, \kappa_{\epsilon}\right)$-structure there is a cocycle $\left[\phi_{s}, \phi_{u}\right] \in \mathrm{TC}_{\Gamma}^{1}\left(X,\left(\operatorname{Spin}^{\mathrm{c}}(n), \kappa_{\epsilon}\right)\right)$ with $\operatorname{Ad}^{c}\left(\left[\phi_{s}, \phi_{u}\right]\right)=\operatorname{Ad}\left(\phi_{s}\right)=\phi$. The cocycle $\left[\phi_{s}, \phi_{u}\right]$ is a lifting by Ad $\times q$ of $\left(\phi, \phi_{u}^{2}\right)$. It remains to check that $\phi_{u}^{2}$ is a cocycle. First, note that $\operatorname{Ad}\left(\partial \phi_{s}\right)=\partial \circ \operatorname{Ad}\left(\phi_{s}\right)=\partial(\phi)=1$. Thus, $\partial \phi_{s}$ takes values in $\operatorname{ker}(\mathrm{Ad})=\mathbb{Z}_{2}$, and

$$
\left(\partial \phi_{s}\right)^{-1}\left(\partial \phi_{u}\right) \in K_{\Gamma}^{2}\left(X,\left(\mathrm{U}(1), \kappa_{\epsilon}\right)\right) \subset K_{\Gamma}^{2}\left(X,\left(\operatorname{Spin}^{\mathrm{c}}(n), \kappa_{\epsilon}\right)\right) .
$$

This cochain is a cocycle as

$$
\left(\partial \phi_{s}\right)^{-1}\left(\partial \phi_{u}\right)=\left[1,\left(\partial \phi_{s}\right)^{-1}\left(\partial \phi_{u}\right)\right]=\left[\partial \phi_{s}, \partial \phi_{u}\right]=\partial\left[\phi_{s}, \phi_{u}\right]=1 .
$$

The cochain $\phi_{u}^{2} \in K_{\Gamma}^{1}\left(X,\left(\mathrm{U}(1), \kappa_{\epsilon}\right)\right)$ is then a cocycle as

$$
\partial\left(\phi_{u}^{2}\right)=\left(\partial \phi_{u}\right)^{2}=\left(\partial \phi_{s}\right)^{-2}\left(\partial \phi_{u}\right)^{2}=\left(\left(\partial \phi_{s}\right)^{-1}\left(\partial \phi_{u}\right)\right)^{2}=1 .
$$

Therefore, the required bundle $L$ can be constructed from $\phi_{u}^{2}$ using [21, Prop. 15].

Proposition 39 can be refined into a statement about cohomology classes. This refinement uses the exact sequences in cohomology obtained by applying [21, Theorem 41] to the two exact sequences of $\Gamma$-groups running diagonally in diagram (2).

Lemma 40. The central exact sequences

$$
\begin{gathered}
1 \rightarrow\left(\mathbb{Z}_{2}, \mathrm{id}_{\epsilon}\right) \rightarrow\left(\operatorname{Spin}(n), \mathrm{id}_{\epsilon}\right) \stackrel{\mathrm{Ad}}{\rightarrow}\left(\mathrm{SO}(n), \mathrm{id}_{\epsilon}\right) \rightarrow 1, \\
1 \rightarrow\left(\mathbb{Z}_{2}, \mathrm{id}_{\epsilon}\right) \rightarrow\left(\mathrm{U}(1), \kappa_{\epsilon}\right) \stackrel{q}{\rightarrow}\left(\mathrm{U}(1), \kappa_{\epsilon}\right) \rightarrow 1,
\end{gathered}
$$

induce the exact sequences

$$
\begin{aligned}
H_{\Gamma}^{1}\left(X,\left(\mathbb{Z}_{2}, \mathrm{id}_{\epsilon}\right)\right) \rightarrow & \mathrm{TC}_{\Gamma}^{1}\left(X,\left(\operatorname{Spin}(n), \mathrm{id}_{\epsilon}\right)\right) \stackrel{\operatorname{Ad}}{\rightarrow} \ldots \\
& \ldots \stackrel{\operatorname{Ad}}{\rightarrow} \mathrm{TC}_{\Gamma}^{1}\left(X,\left(\mathrm{SO}(n), \mathrm{id}_{\epsilon}\right)\right) \stackrel{\Delta_{s}}{\rightarrow} H_{\Gamma}^{2}\left(X,\left(\mathbb{Z}_{2}, \mathrm{id}_{\epsilon}\right)\right), \\
H_{\Gamma}^{1}\left(X,\left(\mathbb{Z}_{2}, \mathrm{id}_{\epsilon}\right)\right) \rightarrow & H_{\Gamma}^{1}\left(X,\left(\mathrm{U}(1), \kappa_{\epsilon}\right)\right) \stackrel{q}{\rightarrow} \ldots \\
& \quad \stackrel{q}{\rightarrow} H_{\Gamma}^{1}\left(X,\left(\mathrm{U}(1), \kappa_{\epsilon}\right)\right) \stackrel{\Delta_{u}}{\rightarrow} H_{\Gamma}^{2}\left(X,\left(\mathbb{Z}_{2}, \mathrm{id}_{\epsilon}\right)\right) .
\end{aligned}
$$


Proposition 39 and Lemma 40 can now be combined to establish an alternative criteria for the existence of a $\left(\operatorname{Spin}^{\mathrm{c}}, \kappa_{\epsilon}\right)$-structure.

Theorem 41. A $\Gamma$-equivariant principal $\mathrm{SO}(n)$-bundle $Q$ with cocycle $\phi$ has a $\left(\mathrm{Spin}^{\mathrm{c}}, \kappa_{\epsilon}\right)$-structure if and only if there exists a cocycle $\psi \in H_{\Gamma}^{1}\left(X,\left(\mathrm{U}(1), \kappa_{\epsilon}\right)\right)$ such that

$$
\Delta_{S}(\phi)=\Delta_{u}(\psi) \in H_{\Gamma}^{2}\left(X,\left(\mathbb{Z}_{2}, \operatorname{id}_{\epsilon}\right)\right)
$$

Proof. Assume that $Q$ has a $\left(\operatorname{Spin}^{\mathrm{c}}, \kappa_{\epsilon}\right)$-structure. By Proposition 39, there exists an cocycle $\left[\phi_{s}, \phi_{u}\right] \in \operatorname{TC}_{\Gamma}^{1}\left(X,\left(\operatorname{Spin}^{\mathrm{c}}(n), \kappa_{\epsilon}\right)\right)$ such that $\phi_{u}^{2}$ is a cocycle and

$$
(\operatorname{Ad} \times q)\left[\phi_{s}, \phi_{u}\right]=\left(\phi_{s}, \phi_{u}^{2}\right) .
$$

As $\left[\phi_{s}, \phi_{u}\right]$ is a cocycle, $\left[\partial \phi_{s}, \partial \phi_{u}\right]=\partial\left[\phi_{s}, \phi_{u}\right]=1$. This implies that $\partial \phi_{s}=\partial \phi_{u}$. Therefore, applying Lemma 40 to $\phi$ and $\phi_{u}^{2}$,

$$
\Delta_{s}(\phi)=\left[\partial \phi_{s}\right]=\left[\partial \phi_{u}\right]=\Delta_{u}\left(\phi_{u}^{2}\right) \in H_{\Gamma}^{2}\left(X,\left(\mathbb{Z}_{2}, \mathrm{id}_{\epsilon}\right)\right) .
$$

Thus, $\psi:=\phi_{u}^{2}$ is the required cocycle.

Conversely, suppose there exists a cocycle $\psi \in H_{\Gamma}^{1}\left(X,\left(\mathrm{U}(1), \kappa_{\epsilon}\right)\right)$ such that

$$
\Delta_{s}(\phi)=\Delta_{u}(\psi) \in H_{\Gamma}^{2}\left(X,\left(\mathbb{Z}_{2}, \mathrm{id}_{\epsilon}\right)\right) .
$$

Then, there are a cochains $\phi_{s}$ with $\operatorname{Ad}\left(\phi_{s}\right)=\phi$, and $\phi_{u}$ with $\phi_{u}^{2}=\psi$ such that

$$
\left[\partial \phi_{s}\right]=\left[\partial \phi_{u}\right] \in K_{\Gamma}^{2}\left(X,\left(\mathbb{Z}_{2}, \mathrm{id}_{\epsilon}\right)\right) .
$$

This implies that $\partial \phi_{s}=\partial \phi^{\prime} \partial \phi_{u}=\partial\left(\phi^{\prime} \phi_{u}\right)$ for some $\phi^{\prime} \in K_{\Gamma}^{1}\left(X,\left(\mathbb{Z}_{2}, \mathrm{id}_{\epsilon}\right)\right)$. Then $\partial\left[\phi_{s}, \phi^{\prime} \phi_{u}\right]=\left[\partial \phi_{s}, \partial\left(\phi^{\prime} \phi_{u}\right)\right]=1$, and $\operatorname{Ad}^{c}\left[\phi_{s}, \phi^{\prime} \phi_{u}\right]=\operatorname{Ad}\left(\phi_{s}\right)=$ $\phi$. Thus, $\left[\phi_{s}, \phi^{\prime} \phi_{u}\right]$ defines a $\left(\operatorname{Spin}^{\mathrm{c}}, \kappa_{\epsilon}\right)$-structure on $Q$.

If $X$ is a manifold acted on by a finite group $H$, and $V \rightarrow X$ is a real $H$-equivariant vector bundle with cocycle $\phi \in \mathrm{TC}_{H}^{1}(X, \mathrm{SO}(n))$, then the obstruction to the existence of an $H$-equivariant Spin-structure on $V$ is the second $\mathbb{Z}_{2}$-valued equivariant Stiefel-Whitney class, which can be defined by $w_{2}^{H}(V):=\Delta_{\text {Spin }}(\phi) \in H_{H}^{2}\left(X, \mathbb{Z}_{2}\right)$. Here $\Delta_{\text {Spin }}(\phi)$ is the connecting map for the exact sequence

$$
H_{H}^{1}\left(X, \mathbb{Z}_{2}\right) \longrightarrow \mathrm{TC}_{H}^{1}(X, \operatorname{Spin}(n)) \stackrel{\mathrm{Ad}}{\longrightarrow} \mathrm{TC}_{H}^{1}(X, \mathrm{SO}(n)) \stackrel{\Delta_{\text {Spin }}}{\longrightarrow} H_{H}^{2}\left(X, \mathbb{Z}_{2}\right),
$$

induced by the central exact sequence

$$
1 \rightarrow \mathbb{Z}_{2} \rightarrow \operatorname{Spin}(n) \stackrel{\mathrm{Ad}}{\rightarrow} \mathrm{SO}(n) \rightarrow 1
$$

If $(\Gamma, \epsilon)$ is the orientifold group defined by $\Gamma:=\mathbb{Z}_{2} \times H$ and $\epsilon(z, h):=$ $z$, then $X$ can be made into an orientifold $\tilde{X}$ for $(\Gamma, \epsilon)$ by trivially 
extending its $H$-action to the $\Gamma$-action $(z, h) x:=h x$. Similarly, the $H$ equivariant vector bundle $V$ can be made into a $\Gamma$-equivariant vector bundle $\tilde{V}$ by trivially extending its $H$-action to the $\Gamma$-action $(z, h) v:=$ $h v$. The cocycle of $\tilde{V}$ is an element $\tilde{\phi} \in \mathrm{TC}_{\Gamma}^{1}\left(X,\left(\mathrm{SO}(n), \mathrm{id}_{\epsilon}\right)\right)$.

In this situation, the quotient map $\pi: \Gamma \rightarrow \Gamma / \mathbb{Z}_{2} \simeq H$ induces a map $\pi: X_{\Gamma}^{\bullet} \rightarrow X_{H}^{\bullet}$ between the simplicial spaces associated to the groups $\Gamma$ and $H$. Because $\pi$ is a homomorphism and satisfies $\pi(\gamma) x=\gamma x$, it commutes with the face maps on these spaces, and defines a pulback map $\pi^{*}$ on cochains. The map $\pi^{*}$ also commutes with the coboundary maps, and provides well-defined extension maps

$$
\pi^{*}: \operatorname{TC}_{H}^{p}(X, G) \rightarrow \operatorname{TC}_{\Gamma}^{p}\left(\tilde{X},\left(G, \mathrm{id}_{\epsilon}\right)\right) \quad \pi^{*}: H_{H}^{p}(X, G) \rightarrow H_{\Gamma}^{p}\left(\tilde{X},\left(G, \mathrm{id}_{\epsilon}\right)\right) .
$$

One then has the following result.

Proposition 42. If $\tilde{V} \rightarrow \tilde{X}$ is the trivial extension of a real H-equivariant vector bundle $V \rightarrow X$, as described above, then

(a) the cocycle for $\tilde{V}$ is the pullback of the cocycle for $V$ by the quotient map $\pi: \Gamma \rightarrow H$,

$$
\tilde{\phi}=\pi^{*} \phi \in H_{\Gamma}^{1}\left(\tilde{X},\left(\mathrm{SO}(n), \mathrm{id}_{\epsilon}\right)\right) .
$$

(b) the second $\mathbb{Z}_{2}$-valued equivariant Stiefel-Whitney class for $V$ satisfies

$$
\pi^{*} w_{2}^{H}(V)=\Delta_{S}\left(\pi^{*} \phi\right) \in H_{\Gamma}^{2}\left(\tilde{X},\left(\mathbb{Z}_{2}, \mathrm{id}_{\epsilon}\right)\right) .
$$

(c) $\tilde{V}$ has a $\left(\operatorname{Spin}^{\mathrm{c}}, \kappa_{\epsilon}\right)$-structure if and only if

$$
\pi^{*} w_{2}^{H}(V)=\Delta_{u}(\psi) \in H_{\Gamma}^{2}\left(\tilde{X},\left(\mathbb{Z}_{2}, \mathrm{id}_{\epsilon}\right)\right),
$$

for some cocycle $\psi \in H_{\Gamma}^{1}\left(\tilde{X},\left(\mathrm{U}(1), \kappa_{\epsilon}\right)\right)$.

Here $\Delta_{s}$ and $\Delta_{u}$ are the connecting maps of Lemma 40.

Proof. If $\left\{s_{a}\right\}$ is a collection of local sections for $V$, then

$$
\pi(z, h) x=h x=(z, h) x \quad \pi(z, h) s_{a}(x)=h s_{a}(x)=(z, h) s_{a}(x),
$$

where $(z, h) \in \Gamma=\mathbb{Z}_{2} \times H, x \in X$. Together with the property which defines the cocycles $\phi$ and $\tilde{\phi}$ [21, Prop. 12], this implies

$$
\begin{array}{r}
s_{b}(\pi(z, h) x) \phi_{b a}(\pi(z, h), x)=\pi(z, h) s_{a}(x)=(z, h) s_{a}(x) \\
=s_{b}((z, h) x) \tilde{\phi}_{b a}((z, h), x) \\
=s_{b}(\pi(z, h) x) \tilde{\phi}_{b a}((z, h), x) .
\end{array}
$$

Thus, $\pi^{*} \phi=\tilde{\phi}$, which proves the the first statement. 
The second statement follows from the existence of the commutative diagram

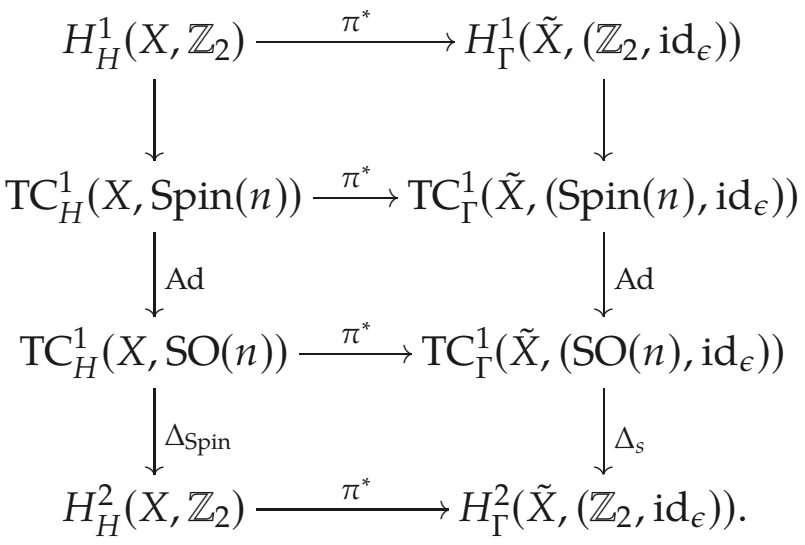

To see that the bottom cell of this diagram commutes, note that if $\psi$ is a lifting of $\phi$, then $\pi^{*} \psi$ is a lifting of $\pi^{*} \phi$. The commutation of $\pi^{*}$ with the coboundary maps then implies

$$
\pi^{*} w_{2}^{H}(V):=\pi^{*} \Delta_{\text {Spin }}(\phi)=\pi^{*} \partial(\psi)=\partial\left(\pi^{*} \psi\right)=\Delta_{S}\left(\pi^{*} \phi\right) .
$$

The third statement follows from the first and second by applying Theorem 41.

To end this section, two important $\left(\operatorname{Spin}^{\mathrm{c}}, \kappa_{\epsilon}\right)$-structures will be described. The first of these is the canonical (Spin $\left.{ }^{\mathrm{C}}, \kappa_{\epsilon}\right)$-structure associated to a real representation $V$ of $\left(\mathbb{Z}_{2}\right.$, id $) \ltimes_{\kappa_{\epsilon}} \operatorname{Spin}^{\mathrm{c}}(n)$. When $\operatorname{dim}(V)=8$, this $\left(\operatorname{Spin}^{\mathrm{c}}, \kappa_{\epsilon}\right)$-structure is used to construct a canonical reduced orientifold spinor bundle over the point orientifold for $\left(\mathbb{Z}_{2}\right.$, id $) \ltimes_{\kappa_{\epsilon}} \operatorname{Spin}^{\mathrm{c}}(n)$, which, in turn, can be used to construct the 8-fold Bott class over $V$ for orientifold $K$-theory [20, Example 4.9]. The second is a canonical $\left(\operatorname{Spin}^{\mathrm{c}}, \kappa_{\epsilon}\right)$-structure on the $n$-sphere. This $\left(\operatorname{Spin}^{\mathrm{c}}, \kappa_{\epsilon}\right)$ structure is used to construct a canonical reduced orientifold spinor bundle on $S^{8 k}$. The reduced orientifold spinor bundle on $S^{8 k}$ can be used to describe the compactification of the 8-fold Bott class over a real representation of $\left(\mathbb{Z}_{2}, \mathrm{id}\right) \ltimes_{\kappa_{\epsilon}} \operatorname{Spin}^{\mathrm{c}}(n)$ [20, Example 4.11]. .

Lemma 43 (The canonical (Spin ${ }^{\mathrm{c}}, \kappa_{\epsilon}$ )-structure over a point). Let $V$ be the representation of $\left(\mathbb{Z}_{2}, \mathrm{id}\right) \ltimes_{\kappa_{\epsilon}} \operatorname{Spin}^{\mathrm{c}}(n)$ on $\mathbb{R}^{n}$ defined by $(\gamma, g) \cdot v:=\operatorname{Ad}^{c}(g) v$. Then

$$
\operatorname{Ad}^{c}: \operatorname{Spin}^{c}(n) \rightarrow \operatorname{SO}(n) \simeq \operatorname{Fr}(V) .
$$

is a $\left(\operatorname{Spin}^{\mathrm{C}}, \kappa_{\epsilon}\right)$-structure for the real equivariant vector bundle $V \rightarrow \mathrm{pt}$ over the point orientifold for $\left(\mathbb{Z}_{2}, \mathrm{id}\right) \ltimes_{\kappa_{\epsilon}} \operatorname{Spin}^{\mathrm{c}}(n)$. 
Proof. The group $\operatorname{Spin}^{\mathrm{c}}(n)$ forms a principal bundle over a point with the trivial projection $\pi(p)=\mathrm{pt}$, and right $\operatorname{Spin}^{c}(n)$ action defined by multiplication. The left action of $\left(\mathbb{Z}_{2}, \mathrm{id}\right) \ltimes_{\kappa_{\epsilon}} \operatorname{Spin}^{\mathrm{c}}(n)$ is taken to be

$$
(\gamma, g) \cdot p:=g \kappa_{\gamma}(p)
$$

for $\gamma \in \Gamma$ and $g, p \in \operatorname{Spin}^{c}(n)$. The inclusion of the conjugation $\kappa$ is the only difference from the corresponding construction in the usual equivariant setting.

Lemma 44 (The canonical $\left(\operatorname{Spin}^{\mathrm{c}}, \kappa_{\epsilon}\right)$-structure on the sphere). The map

$$
\operatorname{Ad}^{c}: \operatorname{Spin}^{c}(n+1) \rightarrow \operatorname{SO}(n+1)
$$

forms a (Spin $\left.{ }^{c}, \kappa_{\epsilon}\right)$-structure for the orientifold $S^{n} \subset \mathbb{R}^{n+1}$ equipped with the action of $\left(\mathbb{Z}_{2}, \mathrm{id}\right) \ltimes_{\kappa_{\epsilon}} \operatorname{Spin}^{\mathrm{c}}(n+1)$ defined by $(\gamma, g) \cdot v:=\operatorname{Ad}^{c}(g) v$.

Proof. In what follows, let $\gamma \in \mathbb{Z}_{2}, g, p \in \operatorname{Spin}^{\mathrm{c}}(n+1), h \in \operatorname{Spin}^{\mathrm{c}}(n)$, $q \in \mathrm{SO}(n+1), f \in \mathrm{SO}(n)$. Also, let $\alpha_{1}: \mathrm{SO}(n) \rightarrow \mathrm{SO}(n+1)$ and $\beta_{1}: \operatorname{Spin}^{\mathrm{C}}(n) \rightarrow \operatorname{Spin}^{\mathrm{C}}(n+1)$ be the maps induced by the inclusion $\mathbb{C l}_{n} \rightarrow \mathbb{C l}_{n+1}$ defined on standard basis elements by $e_{k} \mapsto e_{k+1}$. Equip

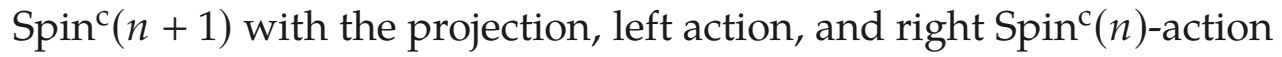

$$
\pi_{s c}(p):=\operatorname{Ad}^{c}(p) e_{1} \quad(\gamma, g) \cdot p:=g \kappa_{\gamma}(p) \quad p \cdot h:=p \beta_{1}(h),
$$

respectively. Again, the presence of the conjugation action $\kappa$ in the left action is the only difference from the corresponding construction in the usual equivariant setting [7, p. 5]. Using the properties of $\kappa$, $\operatorname{Ad}^{c}$ and $\beta_{1}$, it is straightforward to check that $\operatorname{Spin}^{c}(n+1)$ forms a $(\Gamma, \epsilon) \ltimes_{\kappa_{\epsilon}} \operatorname{Spin}^{\mathrm{c}}(n+1)$-semi-equivariant principal $\left(\operatorname{Spin}^{\mathrm{c}}(n), \kappa_{\epsilon}\right)$-bundle,

$$
\begin{array}{r}
\pi_{s c}((\gamma, g) \cdot p)=\pi(g(\gamma p)) \\
=\operatorname{Ad}^{c}(g(\gamma p)) e_{1}=\operatorname{Ad}^{c}(g) \operatorname{Ad}^{c}(\gamma p) e_{1} \\
=\operatorname{Ad}^{c}(g) \operatorname{Ad}^{c}(p) e_{1}=(\gamma, g) \pi_{s c}(p), \\
(\gamma, g) \cdot(p \cdot h)=(\gamma, g) \cdot\left(p \beta_{1}(h)\right) \\
=g\left(\gamma\left(p \beta_{1}(h)\right)\right)=g(\gamma p)\left(\gamma \beta_{1}(h)\right) \\
=g(\gamma p) \beta_{1}(\gamma h)=((\gamma, g) p) \cdot(\gamma h) .
\end{array}
$$

Next, equip $\mathrm{SO}(n+1)$ with the projection, left action, and right $\mathrm{SO}(n)$ action defined by

$$
\pi_{s o}(q):=q e_{1} \quad(\gamma, g) \cdot q:=\operatorname{Ad}^{c}(g) q \quad q \cdot f:=q \alpha_{1}(f),
$$


respectively. It can then be checked that $\mathrm{SO}(n+1)$ forms a $\left(\mathbb{Z}_{2}\right.$, id $) \ltimes_{\kappa_{\epsilon}}$ $\operatorname{Spin}^{c}(n+1)$-equivariant principal SO(n)-bundle,

$$
\begin{aligned}
& \pi_{s o}((\gamma, g) \cdot q)=\pi_{s o}\left(\operatorname{Ad}^{c}(g) q\right)=\operatorname{Ad}^{c}(g) q e_{1}=(\gamma, g) \pi(q), \\
& (\gamma, g) \cdot(q \cdot f)=(\gamma, g) \cdot\left(q \alpha_{1}(f)\right)=\operatorname{Ad}(g) q \alpha_{1}(f)=((\gamma, g) \cdot q) \cdot f .
\end{aligned}
$$

That $\mathrm{Ad}^{c}$ is a semi-equivariant lifting can be checked directly by verifying compatibility with projections, right actions, and left actions,

$$
\begin{gathered}
\pi_{s c}(p)=\operatorname{Ad}^{c}(p) e_{1}=\pi_{s o} \circ \operatorname{Ad}^{c}(p), \\
\operatorname{Ad}^{c}(p \cdot h)=\operatorname{Ad}^{c}\left(p \beta_{1}(h)\right) \\
=\operatorname{Ad}^{c}(p) \operatorname{Ad}^{c}\left(\beta_{1}(h)\right)=\operatorname{Ad}^{c}(p) \alpha_{1}\left(\operatorname{Ad}^{c}(h)\right) \\
=\operatorname{Ad}^{c}(p) \cdot \operatorname{Ad}^{c}(h), \\
\operatorname{Ad}^{c}((\gamma, g) \cdot p)=\operatorname{Ad}^{c}(g(\gamma p)) \\
=\operatorname{Ad}^{c}(g) \operatorname{Ad}^{c}(\gamma p)=\operatorname{Ad}^{c}(g) \operatorname{Ad}^{c}(p) \\
=(\gamma, g) \cdot \operatorname{Ad}^{c}(p) .
\end{gathered}
$$

It remains to check that $\mathrm{SO}(n+1)$ with the given action of $\left(\mathbb{Z}_{2}, \mathrm{id}\right) \ltimes_{\kappa_{\epsilon}}$ $\operatorname{Spin}^{c}(n+1)$ is isomorphic to the equivariant principal $\mathrm{SO}(n)$-bundle $\operatorname{Fr}\left(S^{n}\right)$. First, identify the tangent space of the $n$-sphere with a subbundle of the tangent space to $\mathbb{R}^{n+1}$,

$T S^{n} \simeq\left\{\left(v_{1}, v_{2}\right) \in \mathbb{R}^{n+1} \times \mathbb{R}^{n+1} \mid\left\|v_{1}\right\|=\left\|v_{2}\right\|=1,\left\langle v_{1}, v_{2}\right\rangle=0\right\} \subset T \mathbb{R}^{n+1}$

The standard action of $\mathrm{SO}(n+1)$ on $\mathbb{R}^{n+1}$ associates a matrix to each element $q \in \mathrm{SO}(n+1)$, which will also be denoted $q$. The columns $q_{i}$ of this matrix determine an orthonormal frame

$$
F(q):=\left\{\left(q_{1}, q_{2}\right), \ldots,\left(q_{1}, q_{n+1}\right)\right\} \in \operatorname{Fr}_{q_{1}}\left(T S^{n}\right) .
$$

In this way, $\mathrm{SO}(n+1)$ can be identified with $\operatorname{Fr}\left(T S^{n}\right)$. This identification is compatible with projections as

$$
\pi_{s o}(q)=q e_{1}=q_{1}=\pi_{T S^{n}}(F(q)) .
$$

Compatibility with right actions follows from the fact that

$$
(q \cdot f)_{j}=\left(q \alpha_{1}(f)\right)_{j}= \begin{cases}q_{1} & \text { for } j=1 \\ \sum_{2 \leq i \leq n+1} q_{i} f_{(i-1)(j-1)} & \text { for } j \geq 2 .\end{cases}
$$

Finally, the left action on $\operatorname{Fr}\left(T S^{n}\right)$ can be characterised by observing that a vector $\left(v_{1}, v\right) \in T S^{n}$ is tangent to the curve $(\cos t) v_{1}+(\sin t) v$ at $t=0$. Acting on this curve by $(\gamma, g) \in\left(\mathbb{Z}_{2}, \mathrm{id}\right) \ltimes_{\kappa_{\epsilon}} \operatorname{Spin}^{\mathrm{c}}(n+1)$ 
produces a new curve $(\cos t)\left(\operatorname{Ad}^{c}(g) v_{1}\right)+(\sin t)\left(\operatorname{Ad}^{c}(g) v\right)$ which has $\left(\operatorname{Ad}^{c}(g) v_{1}, \operatorname{Ad}^{c}(g) v\right)$ as its tangent vector at $t=0$. Thus,

$$
(\gamma, g) F(q)=F\left(\operatorname{Ad}^{c}(g) q\right)=F((\gamma, g) q)
$$

and the identification of $\mathrm{SO}(n+1)$ and $\operatorname{Fr}\left(T S^{n}\right)$ is compatible with the left actions.

\section{Dirac Operators on Orientifolds}

In this section, Dirac operators are constructed for orientifolds. By applying a semi-equivariant associated bundle construction with a Clifford module as the model fibre, it is possible to construct spinor bundles with orientifold actions. Both a total spinor bundle, with a right action of $\left(\mathbb{C l}_{n}, \kappa_{\epsilon}\right)$, and a reduced spinor bundle, with the complexification of an irreducible $\mathrm{Cl}_{8 k}$-module as a model fibre, are defined. As in the usual setting, the sections of orientifold spinor bundles are acted on by sections of a Clifford bundle. This action is compatible with the orientifold action on the spinor bundle and a canonical orientifold action on the complex Clifford bundle. In order to construct a Dirac operator on an orientifold, it is neccesary to have a connection which is compatible with Clifford multiplication on sections and the orientifold action. Such a connection can be constructed using results on semi-equivariant connection forms from §A.2. After equipping the orientifold spinor bundles with compatible connections, the orientifold Dirac operator and its reduced counterpart will be defined.

3.1. Orientifold Spinor Bundles. The model fibre of an orientifold spinor bundle is a Clifford module that is semi-equivariant with respect to the action of $\left(\operatorname{Spin}^{c}(n), \kappa_{\epsilon}\right)$. Such modules can be constructed by complexifying $\mathrm{Cl}_{n}$-modules. The main $\mathrm{Cl}_{n}$-modules of interest are $\mathrm{Cl}_{n}$, considered as a module over itself, and the irreducible $\mathrm{Cl}_{8 k}$-modules. $\mathrm{Up}$ to equivalence, there is only one irreducible $\mathrm{Cl}_{8 k}$-module [23, p. 33]. A representative of this equivalence class will be denoted by $\Delta$. Denote the complexifications of these, equipped with their associated orientifold actions, by

$$
\left(\Delta_{c}, \kappa_{\epsilon}\right):=\left(\Delta \otimes \mathbb{C}, \mathrm{id} \otimes \kappa_{\epsilon}\right) \quad\left(\mathbb{C l}_{n}, \kappa_{\epsilon}\right):=\left(\mathrm{Cl}_{n} \otimes \mathbb{C}, \mathrm{id} \otimes \kappa_{\epsilon}\right) .
$$

It is important to note that the complexification $\Delta \otimes \mathbb{C}$ is an irreducible module for $\mathbb{C l}_{8 k}$. This is a non-trivial fact that depends on the representation theory of Clifford algberas.

The orientifold spinor bundles can now be defined by applying the semi-equivariant associated bundle construction to a semi-equivariant 
principal bundle coming from a $\left(\operatorname{Spin}^{\mathrm{c}}, \kappa_{\epsilon}\right)$-structure and a complex Clifford module equipped with an orientifold action.

Definition 45. Let $P \rightarrow \operatorname{Fr}(V)$ be an orientifold-Spin ${ }^{\mathrm{c}}$-structure, and define the following orientifold bundles:

The orientifold spinor bundle

$$
\begin{array}{ll}
\text { The orientifold spinor bundle } & \phi:=P \times_{\left(\operatorname{Spin}^{\mathrm{c}}(n), \kappa_{\epsilon}\right)}\left(\mathbb{C l}_{n}, \kappa_{\epsilon}\right), \\
\text { The reduced orientifold spinor bundle } & \phi:=P \times_{\left(\operatorname{Spin}^{\mathrm{c}}(n), \kappa_{\epsilon}\right)}\left(\Delta_{c}, \kappa_{\epsilon}\right) .
\end{array}
$$

Note that if one disregards the orientifold action, then an orientifold spinor bundle is a complex spinor bundle in the usual sense. In the case of the reduced orientifold spinor bundle, $\Delta_{c}$ is an irreducible module for $\mathrm{Cl}_{8 k}$, as mentioned above. This implies that, disregarding the orientifold action, the reduced orientifold spinor bundle is a reduced complex spinor bundle.

Example 46 (The canonical reduced orientifold spinor bundle over a point). Using Lemma 43 it is possible to construct a $\left(\operatorname{Spin}^{\mathrm{C}}, \kappa_{\epsilon}\right)$ structure $P \rightarrow \operatorname{Fr}(V)$, for the adjoint representation $V$ of $\left(\mathbb{Z}_{2}\right.$, id $) \ltimes_{\kappa_{\epsilon}}$ $\operatorname{Spin}^{\mathrm{c}}(n)$. If $\operatorname{dim}(V)=8 k$, then the irreducible $\mathrm{Cl}_{n}$-module $\Delta$ can be used to construct a canonical reduced spinor bundle $\$ \rightarrow$ pt over the point orientifold.

Example 47 (The canonical reduced orientifold spinor bundle over $S^{8 k}$ ). By Lemma 44 , each sphere $S^{n}$ has a canonical $\left(\mathbb{Z}_{2}\right.$, id $) \ltimes_{\mathcal{K}_{\epsilon}} \operatorname{Spin}^{\mathrm{c}}(n)$ equivariant $\left(\operatorname{Spin}^{\mathrm{C}}, \kappa_{\epsilon}\right)$-structure. If $\operatorname{dim}(V)=8 k$, then the irreducible $\mathrm{Cl}_{n}$-module $\Delta$ can be used to construct a canonical reduced spinor bundle $\$ \rightarrow S^{8 k}$ over the 8-dimensional sphere. This construction is an adaptation, to the orientifold setting, of the Real equivariant spinor bundle defined on $S^{8 k}$ by Atiyah [2, p. 128].

The space of sections of the orientifold spinor bundle carries an action by sections of an orientifold Clifford bundle $\mathbb{C l}(V)$ called Clifford multiplication. When a $\left(\operatorname{Spin}^{\mathrm{c}}, \kappa_{\epsilon}\right)$-structure $P \rightarrow \operatorname{Fr}(V)$ exists, the orientifold Clifford bundle can be expressed as an associated bundle

$$
\mathbb{C l}(V):=P \times_{\left(\operatorname{Spin}^{c}(n), \kappa_{\epsilon}\right)}^{\operatorname{Ad}^{c}}\left(\mathbb{C l}_{n}, \kappa_{\epsilon}\right)
$$

of $P$, and this characterisation can be used to define Clifford multiplication on sections of the associated spinor bundle. Clifford multiplication on sections is defined in terms of the action of $\mathbb{C l}_{n}$ on the model fibre. In order for Clifford multiplication on sections to be well-defined, this fibrewise definition of Clifford multiplication must be compatible with the global topology of the base space. In the orientifold setting, Clifford 
multiplication is also required to be compatible with an orientifold action on the spinor bundle, and a canonical orientifold action on $\mathbb{C l}(V)$. The (Spin $\left.{ }^{c}, \kappa_{\epsilon}\right)$-structure used to construct an orientifold spinor bundle ensures that both of these requirements are fulfilled. Thus, the benefit of working on semi-equivariance and $\left(\mathrm{Spin}^{\mathrm{c}}, \kappa_{\epsilon}\right)$-orientiation is finally observed. In what follows, consider sections of associated bundles to be represented by equivariant maps from the principal bundle $P$ of an underlying $\left(\operatorname{Spin}^{\mathrm{C}}, \kappa_{\epsilon}\right)$-structure $P \rightarrow \operatorname{Fr}(V)$ into the semi-equivariant fibre, as in Lemma 60.

Proposition 48. Sections $\varphi \in \Gamma(\mathbb{C l}(V))$ of the orientifold Clifford bundle act from the left on the sections $\psi \in \Gamma(\phi)$ of the orientifold spinor bundle by

$$
(\varphi \psi)(p)=\varphi(p) \psi(p)
$$

This action is well-defined and satisfies $\gamma(\varphi \psi)=(\gamma \varphi)(\gamma \psi)$.

Proof. Multiplication is well-defined, as

$$
\begin{aligned}
& (\varphi \psi)(p g)=\varphi(p g) \psi(p g) \\
& \quad=\left(g^{-1} \varphi(p) g\right)\left(g^{-1} \psi(p)\right)=g^{-1} \varphi(p) \psi(p)=g^{-1}(\varphi \psi)(p) .
\end{aligned}
$$

Compatibility with the orientifold actions is verified using Lemma 60,

$$
\begin{aligned}
(\gamma(\varphi \psi))(p)=\gamma(\varphi \psi)\left(\gamma^{-1} p\right) & \\
=\gamma\left(\varphi\left(\gamma^{-1} p\right) \psi\left(\gamma^{-1} p\right)\right) & =\left(\gamma \varphi\left(\gamma^{-1} p\right)\right)\left(\gamma \psi\left(\gamma^{-1} p\right)\right) \\
=(\gamma \varphi)(p)(\gamma \psi)(p) & =((\gamma \varphi)(\gamma \psi))(p) .
\end{aligned}
$$

Sections of the orientifold Clifford bundle act on sections of the reduced orientifold spinor bundle in the same way. One can also check that the Clifford multiplication between sections of the orientifold Clifford bundle is well-defined and compatible with the orientifold action.

Because the orientifold spinor bundle has $\left(\mathbb{C l}_{n}, \kappa_{\epsilon}\right)$ as its model fibre, it carries a right action by elements of $\mathbb{C l}_{n}$. This right action is sometimes described as a multigrading [15, pp. 379-380].

Proposition 49. An element $\varphi \in \mathbb{C l}_{n}$ acts from the right on sections $\psi \in$ $\Gamma(\not) b y$

$$
(\psi \varphi)(p)=\psi(p) \varphi .
$$

For $\gamma \in \Gamma$, this action satisfies $\gamma(\psi \varphi)=(\gamma \psi)(\gamma \varphi)$. 
Proof. Consider $\varphi$ as a constant section of the trivial orientifold bundle $P \times_{(G, \theta)}^{\text {id }}\left(\mathbb{C l}_{n}, \kappa_{\epsilon}\right)$. The right action is well-defined,

$$
(\psi \varphi)(p g)=\psi(p g) \varphi(p g)=g^{-1} \psi(p) \varphi(p)=g^{-1}(\psi \varphi)(p) .
$$

It is also compatible with the orientifold actions,

$$
\begin{aligned}
(\gamma(\psi \varphi))(p)=\gamma(\psi \varphi) & \left(\gamma^{-1} p\right) \\
=\gamma & \left(\psi\left(\gamma^{-1} p\right) \varphi\left(\gamma^{-1} p\right)\right)=\left(\gamma \psi\left(\gamma^{-1} p\right)\right)\left(\gamma \varphi\left(\gamma^{-1} p\right)\right) \\
=(\gamma \psi)(p)(\gamma \varphi)(p) & =((\gamma \psi)(\gamma \varphi))(p) .
\end{aligned}
$$

Similar considerations show that there is also a right action of $\mathbb{C l}_{n}$ on $\mathrm{Cl}(V)$ which is compatible with their orientifold actions.

3.2. Connections in Orientifold Spinor Bundles. In order to define an orientifold Dirac operator, a semi-equivariant connection 1-form is needed for the semi-equivariant principal $\left(\operatorname{Spin}^{c}(n), \kappa_{\epsilon}\right)$-bundle $P$ of the (Spin ${ }^{\mathrm{C}}, \kappa_{\epsilon}$ )-structure $P \rightarrow Q$ underlying the orientifold spinor bundle. Such a form can be obtained by using Proposition 39 to extend the lifting $\varphi: P \rightarrow Q$ to a lifting $P \rightarrow Q \times_{X} L$, where $L$ is a semi-equivariant principal $\left(\mathrm{U}(1), \kappa_{\epsilon}\right)$-bundle. A semi-equivariant connection form can then be constructed on $Q \times_{X} L$, using the averaging process of Proposition 62 , and lifted to $P$, using the relationship between the Lie algebras $\mathfrak{s p i n t}^{\mathfrak{c}}(n)$ and $\mathfrak{s v}(n) \oplus \mathfrak{u}(1)$. In the next proposition, $q$ denotes the square map of Diagram (2).

Proposition 50. The map

$$
\left(\operatorname{Ad}^{c} \times q\right)_{*}: \mathfrak{s p i n}^{\mathfrak{c}}(n)=\mathfrak{s p i n}(n) \oplus \mathfrak{u}(1) \rightarrow \mathfrak{s o}(n) \oplus \mathfrak{u}(1)
$$

is an isomorphism, and satisfies

$$
\left(\operatorname{Ad}^{c} \times q\right)_{*} \circ\left(\mathrm{id} \times \kappa_{\epsilon}\right)_{*}=\left(\mathrm{id} \times \kappa_{\epsilon}\right)_{*} \circ\left(\operatorname{Ad}^{c} \times q\right)_{*} .
$$

Proof. That $\left(\operatorname{Ad}^{c} \times q\right) *$ is an isomorphism is a standard result [10, p. 18$20,29]$. The isomorphism can be written down explicitly by making the following identifications

(a) $\mathfrak{s} \mathfrak{D}(n)$ can be identified with the real $n \times n$ skew-symmetric matricies. A basis for the skew-symmetric matricies is defined by $\left\{E_{i j} \mid 1 \leq i<j \leq n\right\}$ where $E_{i j}$ is the $n \times n$ matrix with all entries equal to 0 except for the $(i, j)$ th and $(j, i)$ th entry, which are equal to 1 and -1 respectively. 
(b) $\operatorname{spin}(n)$ can be identified with the linear subspace $\Lambda^{2} \subset \mathrm{Cl}_{n}$ spanned by the elements $\left\{e_{i} e_{j} \mid 1 \leq i<j \leq n\right\}$, see [10, p. 18].

(c) $\mathfrak{u}(1)$ can be identified with $\mathbb{R}$.

With these identifications, $\left(\operatorname{Ad}^{c} \times q\right)_{*}$ is the map

$$
\begin{aligned}
\left(\operatorname{Ad}^{c} \times q\right)_{*}: \mathfrak{s p i n t}(n) \oplus \mathfrak{u}(1) & \rightarrow \mathfrak{s v}(n) \oplus \mathfrak{u}(1) \\
\left(e_{i} e_{j}, t\right) & \mapsto\left(2 E_{i j}, 2 t\right),
\end{aligned}
$$

see $[10$, pp. 19-20,29]. Also, the $\Gamma$-actions on $\mathfrak{s p i n}(n) \oplus \mathfrak{u}(1)$ and $\mathfrak{s} \mathfrak{p}(n) \oplus$ $\mathfrak{u}(1)$ are

$$
\begin{aligned}
\left(\operatorname{id} \oplus \mathcal{K}_{\epsilon}\right)_{*}: \operatorname{spin}(n) \oplus \mathfrak{u}(1) & \rightarrow \mathfrak{s p i n}(n) \oplus \mathfrak{u}(1) \\
\left(e_{i} e_{j}, t\right) & \mapsto\left(e_{i} e_{j}, \iota_{\epsilon}(t)\right) \\
\left(\operatorname{id} \oplus \kappa_{\epsilon}\right)_{*}: \mathfrak{s D}(n) \oplus \mathfrak{u}(1) & \rightarrow \mathfrak{s o}(n) \oplus \mathfrak{u}(1) \\
\left(E_{i j}, t\right) & \mapsto\left(E_{i j}, \iota_{\epsilon}(t)\right),
\end{aligned}
$$

where $\iota_{\epsilon}: \mathbb{R} \rightarrow \mathbb{R}$ is the involutive action induced by $\iota: t \mapsto-t \in \mathbb{R}$. Examining these maps, it is clear that $\left(\operatorname{Ad}^{c} \times q\right)_{*} \circ\left(\mathrm{id} \times \kappa_{\epsilon}\right)_{*}=(\mathrm{id} \times$ $\left.\kappa_{\epsilon}\right)_{*} \circ\left(\operatorname{Ad}^{c} \times q\right)_{*}$.

Proposition 51. Let $\varphi_{Q}: P \rightarrow Q$ be a $\left(\operatorname{Spin}^{\mathrm{c}}, \kappa_{\epsilon}\right)$-structure. The semiequivariant principal bundle $P$ carries a $\Gamma$-semi-equivariant connection 1form.

Proof. By Proposition 39, there exists a lifting

$$
\varphi_{Q} \times \varphi_{L}: P \rightarrow Q \times_{X} L
$$

by $\operatorname{Ad}^{c} \times q$, where $L$ is a semi-equivariant principal $\left(\mathrm{U}(1), \kappa_{\epsilon}\right)$-bundle. The equivariant principal bundle $Q$ has an equivariant connection 1form $\omega_{Q}: T Q \rightarrow \mathfrak{s p}(n)$ determined by an equivariant metric. The semi-equivariant principal bundle $L$ has a semi-equivariant connection 1 -form $\omega_{L}: T L \rightarrow \mathfrak{u}(1)$ constructed by applying Proposition 62 to any choice of connection 1-form for $L$. Together, these two connection 1 -forms define a semi-equivariant connection 1-form

$$
\omega_{Q} \oplus \omega_{L}: T\left(Q \times_{X} L\right) \rightarrow \mathfrak{s} \mathfrak{D}(n) \oplus \mathfrak{u}(1) .
$$

Using the $\left(\operatorname{Spin}^{\mathrm{c}}, \kappa_{\epsilon}\right)$-structure $\varphi$ and Proposition 50, the connection 1-form $\omega_{Q} \oplus \omega_{L}$ can be lifted to a connection 1-form

$$
\begin{aligned}
\omega: T P & \rightarrow \operatorname{spin}^{c}(n) \\
v & \mapsto\left(\operatorname{Ad}^{c} \times q\right)_{*}^{-1} \circ\left(\omega_{Q} \oplus \omega_{L}\right) \circ\left(\varphi_{Q} \times \varphi_{L}\right)_{*}(v) .
\end{aligned}
$$

The semi-equivariance of $\omega$ follows from the semi-equivariance of $\omega_{Q} \oplus$ $\omega_{L}$, and the equivariance of $\left(\varphi_{Q} \times \varphi_{L}\right)_{*}$ and $\left(\operatorname{Ad}^{c} \times q\right)_{*}$. 
The next proposition shows that the connection 1-form constructed by Proposition 51 defines a covariant derivative on the orientifold spinor bundle that is equivariant with respect to the action of $\Gamma$. In this proposition, sections will be considered as maps $\psi: P \rightarrow \mathbb{C l}_{n}$ satisfying $\psi(g p)=g^{-1} \psi(p)$, and will be acted on by the $\Gamma$-action defined in Lemma 60. From the point of view of the exterior covariant derivative, these maps are order-zero tensorial forms $\psi \in \Lambda^{0}\left(P, \mathbb{C l}_{n}\right)$. For the details of tensorial forms and exterior covariant derivatives, see [10, §B.3-4] [22, §II.5].

Proposition 52. Let $\varphi: P \rightarrow Q$ be a $\left(\operatorname{Spin}^{\mathrm{c}}, \kappa_{\epsilon}\right)$-structure. The semiequivariant connection 1-form $\omega$, defined on P by Proposition 51, determines an exterior covariant derivative

$$
d^{\omega}: \Lambda^{0}\left(P, \mathbb{C l}_{n}\right) \rightarrow \Lambda^{1}\left(P, \mathbb{C l}_{n}\right)
$$

that satisfies the condition

$$
d^{\omega}\left(\kappa_{\epsilon(\gamma)} \circ \psi \circ \eta_{\gamma^{-1}}\right)=\kappa_{\epsilon(\gamma)} \circ d^{\omega} \psi \circ\left(\eta_{\gamma^{-1}}\right)_{* \prime}
$$

where $\psi \in \Lambda^{0}\left(P, \mathbb{C l}_{n}\right)$, $\eta$ is the $\Gamma$-action on $P$, and $\kappa_{\epsilon}$ is the conjugation action on $\mathrm{Cl}_{n}$.

Proof. The vertical projection associated to the connection form $\omega$ is defined by

$$
\left.\pi_{V}\right|_{p}:=\left(R^{p}\right)_{*} \circ \omega: T P_{p} \rightarrow T P_{p} .
$$

Therefore, the exterior covariant derivative can be written as

$d^{\omega} \psi(v)=d \psi \circ \pi_{H}(v)=d \psi(v)-d \psi \circ \pi_{V}(v)=d \psi(v)-d \psi \circ\left(R^{p}\right)_{*} \circ \omega(v)$,

where $v \in T P_{p}, \psi \in \Lambda^{0}\left(P, \mathbb{C l}_{n}\right)$, and $\pi_{H}$ is the horizontal projection. The first term of the decomposition (5) is equivariant, as the properties of the exterior derivative imply that

$$
d\left(\kappa_{\epsilon(\gamma)} \circ \psi \circ \eta_{\gamma^{-1}}\right)=\kappa_{\epsilon(\gamma)} \circ d \psi \circ\left(\eta_{\gamma^{-1}}\right)_{*} .
$$

The semi-equivariance of $P$ implies the identity $\left(\eta_{\gamma}\right)_{*} \circ\left(R^{p}\right)_{*}=\left(R^{\gamma p}\right)_{*} \circ$ $\left(\theta_{\gamma}\right)_{*}$. Together with the the semi-equivariance of $\omega$, this implies that

$$
\begin{aligned}
d\left(\kappa_{\epsilon(\gamma)} \circ \psi \circ \eta_{\gamma^{-1}}\right) \circ\left(R^{p}\right)_{*} \circ \omega & =\kappa_{\epsilon(\gamma)} \circ d \psi \circ\left(\eta_{\gamma^{-1}}\right)_{*} \circ\left(R^{p}\right)_{*} \circ \omega \\
& =\kappa_{\epsilon(\gamma)} \circ d \psi \circ\left(R^{\gamma^{-1} p}\right)_{*} \circ\left(\theta_{\gamma^{-1}}\right)_{*} \circ \omega \\
& =\kappa_{\epsilon(\gamma)} \circ d \psi \circ\left(R^{\gamma^{-1} p}\right)_{*} \circ \omega \circ\left(\eta_{\gamma^{-1}}\right)_{*} .
\end{aligned}
$$

Therefore, the second term of the decomposition (5) is also equivariant. 
Proposition 52 applies equally well to the reduced orientifold spinor bundle if $\mathbb{C l}_{n}$ is replaced with $\Delta_{c}$.

As in the non-equivariant case, the exterior covariant derivative is also equivariant with respect to the right action of $\mathbb{C l}_{n}$ on the orientifold spinor bundle.

Proposition 53. Let $\varphi: P \rightarrow Q$ be a $\left(\operatorname{Spin}^{\mathrm{c}}, \kappa_{\epsilon}\right)$-structure. The semiequivariant connection 1-form $\omega$, defined on P by Proposition 51, determines an exterior covariant derivative

$$
d^{\omega}: \Lambda^{0}\left(P, \mathbb{C l}_{n}\right) \rightarrow \Lambda^{1}\left(P, \mathbb{C l}_{n}\right)
$$

that satisfies

$$
d^{\omega}(\psi \varphi)=d^{\omega}(\psi) \varphi,
$$

for $\psi \in \Lambda^{0}\left(P, \mathbb{C l}_{n}\right)$ and $\varphi \in \mathbb{C l}_{n}$.

3.3. Dirac Operators on Orientifolds. At this stage, all of the preliminary constructions have been completed. It is now possible to construct the orientifold Dirac operator and reduced orientifold Dirac operator.

Definition 54. Let $\nabla^{L}$ denote the connection associated to a $\left(\operatorname{Spin}^{\mathrm{c}}, \kappa_{\epsilon}\right)$ structure $P \rightarrow \operatorname{Fr}(T M)$ by Proposition 51, and $\mu$ denote Clifford multiplication by sections of $T^{*} M \simeq T M \subset \mathbb{C l}(T M)$. Define the orientifold Dirac operator and reduced orientifold Dirac operator, respectively, by

$$
\begin{aligned}
& \not D:=\mu \circ \nabla^{L}: \Gamma(\not) \rightarrow \Gamma\left(T^{*} M \otimes \not\right) \rightarrow \Gamma(\not), \\
& \not D:=\mu \circ \nabla^{L}: \Gamma(\$) \rightarrow \Gamma\left(T^{*} M \otimes \$\right) \rightarrow \Gamma(\$) .
\end{aligned}
$$

The orientifold Dirac operator and reduced orientifold Dirac operator are complex Dirac operators, in the usual sense. However, they are equivariant with respect to the orientifold actions on their spinor bundles. Thus, when $\epsilon: \Gamma \rightarrow \mathbb{Z}_{2}$ is non-trivial, they have anti-linear symmetries.

Proposition 55. The orientifold Dirac operator is equivariant with respect to the left action of $\Gamma$ on sections of $\phi$,

$$
\mathscr{D}(\gamma \psi)=\gamma \mathscr{D}(\psi)
$$

Proof. This follows from Propositions 48 and 52.

The same arguments show that the reduced orientifold spinor bundle is also $\Gamma$-equivariant. In addition to $\Gamma$-equivariance, the orientifold Dirac operator is equivariant with respect to the right action of $\left(\mathbb{C l}_{n}, \kappa_{\epsilon}\right)$ on the orientifold spinor bundle. 
Proposition 56. The orientifold Dirac operator is equivariant with respect to the right action of $\mathbb{C l}_{n}$ on sections of $\phi$,

$$
\mathscr{D}(\psi \varphi)=\mathscr{D}(\psi) \varphi \text {. }
$$

Proof. This follows from Propositions 49 and 53.

Note, in particular, that left and right equivariance together imply that the index of $\mathscr{D}$ consists of vector spaces which are both Clifford modules and orientifold representations of $(\Gamma, \epsilon)$.

The main aim of this paper is now complete, and the following theorem has been proved.

Theorem 57. Let $X$ be an orientifold with orientifold group $(\Gamma, \epsilon)$.

(a) If $W_{3}^{(\Gamma, \epsilon)}(X)=0$, then $X$ carries an orientifold Dirac operator.

(b) If $W_{3}^{(\Gamma, \epsilon)}(X)=0$ and $\operatorname{dim}(X)=8$, then $X$ carries a reduced orientifold Dirac operator.

In particular, if $X$ is an $8 k$-dimensional Real manifold and $W_{3}^{\left(\mathbb{Z}_{2}, \mathrm{id}\right)}(X)=0$, then $X$ carries a reduced Real Dirac operator.

\section{Related Work and Applications}

To put the construction of the orientifold Dirac operator in context, it is worth breifly recalling the position that Spin-structures and Spin-Dirac operators occupy in the K-theory of real vector bundles. The centrality of Spin-structures and the Spin-Dirac operator in the K-theory of real vector bundles stems from their role in Atiyah's index theoretic proof of the Thom isomorphism theorem [2], which provides an isomorphism $\mathrm{KO}(\mathrm{X}) \rightarrow \mathrm{KO}(\mathrm{V})$ between the $\mathrm{KO}$-theory of a manifold and any $8 k$-dimensional Spin-oriented real vector bundle $V \rightarrow X$. The proof proceeds by compactifying the fibres of $V$ into a family of $8 k$-dimensional spheres, each of which is equipped with a canonical reduced Dirac operator. The families index map $K O(V) \rightarrow K O(X)$ associated to this family of Dirac operators is then shown to provide an inverse to the Thom map. This method of proof naturally accomodates additional symmetries, provided that the appropriate analogue of Spin-structure and Dirac operator can be determined. In this way, Atiyah was able to prove the equivariant Thom isomorphism [2]. The $\left(\operatorname{Spin}^{\mathrm{C}}, \kappa_{\epsilon}\right)$-structures and orientifold Dirac operators defined in this paper play an exactly analogous role in the K-theory of orientifold bundles. These allow Atiyah's argument to be extended, providing 
a proof of the corresponding Thom isomorphism theorem for orientifold K-theory [20, Theorem 4.30]. They also provide a basis for the definition of geometric orientifold K-homology [20, Chapter 6].

Orientation conditions in $K$-theory, such as (Spin $\left.{ }^{\mathrm{C}}, \kappa_{\epsilon}\right)$-orientibility, are closely related to the topic of twisted K-theory. The twisted Ktheory of Real topological groupoids has been studied by Moutuou using a Čech cohomology for Real groupoids [25]. In a more algebraic context, Karoubi and Weibel have studied an equivariant twisted $K$-theory that includes KR-theory as a special case [18]. Another approach by Hekmati et al. [14], motivated by applications to orientifold string theories, studies $K R$-orientiation and twisting using a Real sheaf cohomology theory. The work of Freed and Moore on topological insulators [9] also treats twistings of K-theory in the presence of symmetry.

Orientifold string theory and the classification of topological insulators are two areas in which the constructions of this paper have potential applications. The connection between the present investigation and string theory begins with the classification of D-brane charges using $K$-theory, as described in $[24,30]$. Results in index theory allow one to pass from $K$-theory to an analytic K-homology theory in which classes are represented by elliptic operators. Each class in this K-homology theory may be represented by a Dirac operator that has been twisted with a vector bundle. By replacing these Dirac operators with classes formed from the Spin ${ }^{\mathrm{C}}$-structures and vector bundles used to construct them, it is possible to define a $K$-homology theory in entirely geometric terms $[5,4,6]$. This characterisation of D-brane charge is of interest, as the geometric data comprising such a K-homology class has physical interpretations [3] [28, §4]. Three types of orientifold string theories are listed in [30, p. 26-27], along with the corresponding $K$-theories that classifying the associated D-brane charges. In the first of these, D-brane charges are classified by KR-theory. The $\left(\operatorname{Spin}^{\mathrm{C}}, \kappa_{\epsilon}\right)$-structure and orientifold Dirac operators constructed in this paper provide the ingredients neccesary to generalise the above discussion to $K R$-theory and the K-theory of orientifold bundles. The two other possibilities listed in [30, p. 26-27] involve K-theory with sign-choice. This K-theory has been studied by Doran et al. [8] using methods from non-commutative geometry. The K-theory with sign-choice is a subgroup of the K-theory orientifold bundles. Many of the constructions discussed in the present paper could be modified to incorporate sign-choice structures.

In recent years, there there has been much interest in the classification of topological insulators. These classification attempts lead naturally to the consideration of topological invariants which respect anti-linear symmetries [17, 11, 12, 13, 26]. Contact with Clifford al- 
gebras and K-theory has been made through the work of Kitaev [19]. Another framework for studying topological insulators, using twisted K-theories, has been described by Freed and Moore [9]. The K-theory of orientifold bundles is a primary example within their framework. Thus, it appears that there is potential for index invariants derived from the orientifold Dirac operator to be applied to the classification of topological insulators. 


\section{A. Semi-equivariant Constructions}

A.1. Semi-equivariance and Associated Bundles. The construction of associated bundles from semi-equivairant principal bundles differs slightly from the corresponding equivariant construction. When forming an equivariant vector bundle from an equivariant principal bundle, the only requirement on the model fibre is that it carries carries an action of the structure group $G$. However, when forming a vector bundle from a semi-equivariant principal bundle, it is neccesary to use a model fibre that carries both an action of the structure group $G$ and an action of the equivariance group $\Gamma$. As on the semi-equivariant principal bundle, these two actions are required to combine into an action of the semi-direct product group $\Gamma \ltimes_{\theta} G$. Although the action of the equivariance group $G$ on the model fibre is required to be linear, the action of the equivariance group $\Gamma$ is not. This makes it possible to construct associated bundles with $\Gamma$-actions that are not linear. In particular, it is possible to construct complex vector bundles equipped with linear/anti-linear actions as semi-equivariant associated bundles.

Definition 58. Let $P$ be a $\Gamma$-semi-equivariant principal $(G, \theta)$-bundle. A semi-equivariant fibre for $P$ is a vector space $V$ equipped with a linear action of $G$ and an action of $\Gamma$ by diffeomorphisms, such that

$$
\gamma(g v)=(\gamma g)(\gamma v)
$$

Definition 59. Let $P$ be a $\Gamma$-semi-equivariant principal $(G, \theta)$-bundle, and $V$ be a semi-equivariant fibre for $P$. The semi-equivariant associated bundle is the vector bundle

$$
P \times{ }_{(G, \theta)} V:=P \times V / \sim
$$

where $(p, v) \sim\left(p g^{-1}, g v\right)$. This bundle carries an action of $\Gamma$ defined by

$$
\gamma(p, v):=(\gamma p, \gamma v)
$$

Note that the $\Gamma$-action on $P \times_{(G, \theta)} V$ is well-defined because

$$
\begin{aligned}
\gamma\left[p g^{-1}, g v\right]=\left[\gamma\left(p g^{-1}\right), \gamma(g v)\right] \\
=\left[(\gamma p)(\gamma g)^{-1},(\gamma g)(\gamma v)\right]=[\gamma p, \gamma v]=\gamma[p, v] .
\end{aligned}
$$

Sections of associated bundles are often represented as equivariant maps from the principal bundle into the model fibre. It is sometimes useful to express the action of $\Gamma$ on a section in this way. 
Lemma 60. Sections of $P \times_{(G, \theta)} V$ are in bijective correspondence with maps $\psi: P \rightarrow V$ such that $\psi(p g)=g^{-1} \psi(p)$. The $\Gamma$-action on sections of $P \times_{(G, \theta)} V$ corresponds to the $\Gamma$-action

$$
(\gamma \psi)(p)=\gamma \psi\left(\gamma^{-1} p\right)
$$

on these maps.

Proof. A map $\psi: P \rightarrow V$ with $\psi(p g)=g^{-1} \psi(p)$ corresponds to the section of $P \times_{(G, \theta)} V$ defined by $s(p):=[p, \psi(p)]$. The $\Gamma$-action on such a section is

$$
\begin{aligned}
& (\gamma s)(p):=\gamma s\left(\gamma^{-1} p\right) \\
& \quad=\gamma\left[\gamma^{-1} p, \psi\left(\gamma^{-1} p\right)\right]=\left[\gamma \gamma^{-1} p, \gamma \psi\left(\gamma^{-1} p\right)\right]=\left[p, \gamma \psi\left(\gamma^{-1} p\right)\right] .
\end{aligned}
$$

Thus, the corresponding map on $P$ is $p \mapsto \gamma \psi\left(\gamma^{-1} p\right)$.

A.2. Semi-equivariant Connections. In the smooth non-equivariant setting, a connection for a principal $G$-bundle $P$ can be expressed as a $\mathfrak{g}$-valued 1-form on the tangent space $T P$, where $\mathfrak{g}$ is the Lie algebra of the structure group $G$ [22, Chapter 2], [10, Appendix B]. A $\Gamma$-semiequivariant $(G, \theta)$-principal bundle $[21, \S 2]$ has a $\Gamma$-group $(G, \theta)$ as its structure group. The differentials $\left(\theta_{\gamma}\right)_{*}$ of the $\Gamma$-action on $G$ form a $\Gamma$-action on the Lie algebra $\mathfrak{g}$. A connection in a semi-equivariant principal bundle must be compatible with this action if it is to produce an equivariant connection in an associated bundle. The definition of a semi-equivariant connection 1-form is given below, along with an averaging proceedure that can be used to construct semi-equivariant connections. In what follows, let $R_{g}(p)=R^{p}(g):=p g$ denote the multiplication maps associated to the right action on a principal Gbundle $P$. Also, let $R_{g}(h):=h g$ denote the right action of $G$ on itself. Note that $\left(R^{p}\right)_{*}\left(A_{e}\right)$ defines the vector field induced on $P$ by an element $A \in \mathfrak{g}$, and the adjoint map on $\mathfrak{g}$ may be expressed as $\operatorname{Ad}_{g^{-1}}=\left(R_{g}\right)_{*}$.

Definition 61. Let $(P, \eta)$ be a smooth $\Gamma$-semi-equivariant principal $(G, \theta)$-bundle with $\Gamma$-action $\eta$, and let $\mathfrak{g}$ be the Lie alegebra of $G$. A $\Gamma$-semi-equivariant connection 1 -form on $P$ is a Lie algebra valued 1 -form

$$
\omega: T P \rightarrow \mathfrak{g}
$$

such that for all $\gamma \in \Gamma, g \in G, A \in \mathfrak{g}$, and $p \in P$,

$$
\omega \circ\left(R^{p}\right)_{*}\left(A_{e}\right)=A \quad \omega \circ\left(R_{g}\right)_{*}=\left(R_{g}\right)_{*} \circ \omega \quad \omega \circ\left(\eta_{\gamma}\right)_{*}=\left(\theta_{\gamma}\right)_{*} \circ \omega .
$$


When $\Gamma$ is finite, a semi-equivariant connection can be constructed from a given connection by a twisted averaging procedure.

Proposition 62. Let $\Gamma$ be a finite Lie group, and suppose that $P$ is a smooth $\Gamma$-semi-equivariant principal $(G, \theta)$-bundle with $\Gamma$-action $\eta$. If $\omega: T P \rightarrow \mathfrak{g}$ is a connection form on $P$, then

$$
\omega_{\Gamma}:=\sum_{\gamma \in \Gamma}\left(\theta_{\gamma}\right)_{*} \circ \omega \circ\left(\eta_{\gamma^{-1}}\right)_{*}
$$

is a $\Gamma$-semi-equivariant connection on $P$.

Proof. First note that, as $\theta$ is an automorphism and $P$ is semi-equivariant, identities are induced between the differentials of the various actions. For $\gamma \in \Gamma, g, h \in G$, and $p \in P$

$$
\begin{aligned}
& \gamma(h g)=(\gamma h)(\gamma g) \Longrightarrow \quad\left(\theta_{\gamma}\right)_{*} \circ\left(R_{g}\right)_{*}=\left(R_{\gamma g}\right)_{*} \circ\left(\theta_{\gamma}\right)_{*} \\
& \gamma(p g)=(\gamma p)(\gamma g) \Longrightarrow\left\{\begin{array}{l}
\left(\eta_{\gamma}\right)_{*} \circ\left(R_{g}\right)_{*}=\left(R_{\gamma g}\right)_{*} \circ\left(\eta_{\gamma}\right)_{*} \\
\left(\eta_{\gamma}\right)_{*} \circ\left(R^{p}\right)_{*}=\left(R^{\gamma p}\right)_{*} \circ\left(\theta_{\gamma}\right)_{*} .
\end{array}\right.
\end{aligned}
$$

To check that $\omega_{\Gamma}$ is a connection, first observe that the condition $\omega_{\Gamma} \circ$ $\left(R^{p}\right)_{*}\left(A_{e}\right)=A$ holds,

$$
\begin{aligned}
\left(\theta_{\gamma}\right)_{*} \circ \omega \circ\left(\eta_{\gamma^{-1}}\right)_{*} \circ\left(R^{p}\right)_{*}\left(A_{e}\right) & =\left(\theta_{\gamma}\right)_{*} \circ \omega \circ\left(R^{\gamma^{-1} p}\right)_{*} \circ\left(\theta_{\gamma^{-1}}\right)_{*}\left(A_{e}\right) \\
& =\left(\theta_{\gamma}\right) \circ \omega \circ\left(R^{\gamma^{-1} p}\right)_{*}\left(\left(\theta_{\gamma^{-1}}\right)_{*}(A)_{e}\right) \\
& =\left(\theta_{\gamma}\right)_{*} \circ\left(\theta_{\gamma^{-1}}\right)_{*}(A) \\
& =A .
\end{aligned}
$$

The condition $\omega_{\Gamma} \circ\left(R_{g}\right)_{*}=\left(R_{g}\right)_{*} \circ \omega_{\Gamma}$ also holds, as

$$
\begin{aligned}
\left(\theta_{\gamma}\right)_{*} \circ \omega \circ\left(\eta_{\gamma^{-1}}\right)_{*} \circ\left(R_{g}\right)_{*} & =\left(\theta_{\gamma}\right)_{*} \circ \omega \circ\left(R_{\gamma^{-1} g}\right)_{*} \circ\left(\eta_{\gamma^{-1}}\right)_{*} \\
& =\left(\theta_{\gamma}\right)_{*} \circ\left(R_{\gamma^{-1} g}\right)_{*} \circ \omega \circ\left(\eta_{\gamma^{-1}}\right)_{*} \\
& =\left(R_{g}\right)_{*} \circ\left(\theta_{\gamma}\right)_{*} \circ \omega \circ\left(\eta_{\gamma^{-1}}\right)_{*} .
\end{aligned}
$$

Finally, semi-equivariance holds, as

$$
\begin{aligned}
\omega_{\Gamma} \circ\left(\eta_{\gamma}\right)_{*} & =\left(\sum_{\gamma_{1} \in \Gamma}\left(\theta_{\gamma_{1}}\right)_{*} \circ \omega \circ\left(\eta_{\gamma_{1}^{-1}}\right)_{*}\right) \circ\left(\eta_{\gamma}\right)_{*} \\
& =\sum_{\gamma_{1} \in \Gamma}\left(\theta_{\gamma_{1}}\right)_{*} \circ \omega \circ\left(\eta_{\gamma_{1}^{-1} \gamma}\right)_{*} \\
& =\sum_{\gamma_{2} \in \Gamma}\left(\theta_{\gamma \gamma_{2}^{-1}}\right)_{*} \circ \omega \circ\left(\eta_{\gamma_{2}}\right)_{*} \\
& =\left(\theta_{\gamma}\right)_{*} \circ\left(\sum_{\gamma_{2} \in \Gamma}\left(\theta_{\gamma_{2}^{-1}}\right)_{*} \circ \omega \circ\left(\eta_{\gamma_{2}}\right)_{*}\right) \\
& =\left(\theta_{\gamma}\right)_{*} \circ \omega_{\Gamma} .
\end{aligned}
$$




\section{REFERENCES}

[1] Emil Artin and John Tate. Class field theory. AMS Chelsea Publishing, Providence, RI, 2009. Reprinted with corrections from the 1967 original.

[2] M. F. Atiyah. Bott periodicity and the index of elliptic operators. Quart. J. Math. Oxford Ser. (2), 19:113-140, 1968.

[3] Paul Baum. K-homology and D-branes. In Superstrings, geometry, topology, and $C^{*}$-algebras, volume 81 of Proc. Sympos. Pure Math., pages 81-94. Amer. Math. Soc., Providence, RI, 2010.

[4] Paul Baum and Ronald G. Douglas. Index theory, bordism, and K-homology. In Operator algebras and K-theory (San Francisco, Calif., 1981), volume 10 of Contemp. Math., pages 1-31. Amer. Math. Soc., Providence, R.I., 1982.

[5] Paul Baum and Ronald G. Douglas. K homology and index theory. In Operator algebras and applications, Part I (Kingston, Ont., 1980), volume 38 of Proc. Sympos. Pure Math., pages 117-173. Amer. Math. Soc., Providence, R.I., 1982.

[6] Paul Baum, Nigel Higson, and Thomas Schick. On the equivalence of geometric and analytic K-homology. Pure Appl. Math. Q., 3(1, part 3):1-24, 2007.

[7] Paul Baum, Nigel Higson, and Thomas Schick. A geometric description of equivariant K-homology for proper actions. In Quanta of maths, volume 11 of Clay Math. Proc., pages 1-22. Amer. Math. Soc., Providence, RI, 2010.

[8] Charles Doran, Stefan Méndez-Diez, and Jonathan Rosenberg. Tduality for orientifolds and twisted KR-theory. Lett. Math. Phys., 104(11):1333-1364, 2014.

[9] D. S. Freed and G. W. Moore. Twisted Equivariant Matter. Annales Henri Poincaré, 14:1927-2023, December 2013.

[10] Thomas Friedrich. Dirac operators in Riemannian geometry, volume 25 of Graduate Studies in Mathematics. American Mathematical Society, Providence, RI, 2000. Translated from the 1997 German original by Andreas Nestke. 
[11] Liang Fu and C. L. Kane. Time reversal polarization and a $Z_{2}$ adiabatic spin pump. Phys. Rev. B, 74:195312, Nov 2006.

[12] Liang Fu and C. L. Kane. Topological insulators with inversion symmetry. Phys. Rev. B, 76:045302, Jul 2007.

[13] Liang Fu, C. L. Kane, and E. J. Mele. Topological insulators in three dimensions. Phys. Rev. Lett., 98:106803, Mar 2007.

[14] Pedram Hekmati, Michael K. Murray, Richard J. Szabo, and Raymond F. Vozzo. Real bundle gerbes, orientifolds and twisted KRhomology. arXiv e-prints, page arXiv:1608.06466, Aug 2016.

[15] Nigel Higson and John Roe. Analytic K-homology. Oxford Mathematical Monographs. Oxford University Press, Oxford, 2000. Oxford Science Publications.

[16] Laurens Jansen and Michael Boon. Theory of finite groups. Applications in physics. (Symmetry groups of quantum mechanical systems.). North-Holland Publishing Co., Amsterdam; Interscience Publishers John Wiley \& Sons, Inc., New York, 1967.

[17] C. L. Kane and E. J. Mele. $Z_{2}$ topological order and the quantum spin hall effect. Phys. Rev. Lett., 95:146802, Sep 2005.

[18] Max Karoubi and Charles Weibel. Twisted K-theory, real Abundles and Grothendieck-Witt groups. J. Pure Appl. Algebra, 221(7):1629-1640, 2017.

[19] A. Kitaev. Periodic table for topological insulators and superconductors. In American Institute of Physics Conference Series, volume 1134, pages 22-30, May 2009.

[20] Simon Kitson. Dirac Operators on Orientifolds. PhD thesis, Australian National University, 2020. Available from the ANU Open Research repository http:/ /hdl.handle.net/1885/201763.

[21] Simon Kitson. A Semi-equivariant Dixmier-Douady Invariant. arXiv:2003.09138v1, 2020.

[22] Shoshichi Kobayashi and Katsumi Nomizu. Foundations of differential geometry. Vol I. Interscience Publishers, a division of John Wiley \& Sons, New York-London, 1963.

[23] H. Blaine Lawson, Jr. and Marie-Louise Michelsohn. Spin geometry, volume 38 of Princeton Mathematical Series. Princeton University Press, Princeton, NJ, 1989. 
[24] Ruben Minasian and Gregory Moore. K-theory and RamondRamond charge. Journal of High Energy Physics, 1997(11):002-002, nov 1997.

[25] El-kaïoum M. Moutuou. On groupoids with involutions and their cohomology. New York J. Math., 19:729-792, 2013.

[26] Xiao-Liang Qi, Taylor L. Hughes, and Shou-Cheng Zhang. Topological field theory of time-reversal invariant insulators. Phys. Rev. B, 78:195424, Nov 2008 .

[27] Richard G. Swan. Vector bundles and projective modules. Trans. Amer. Math. Soc., 105:264-277, 1962.

[28] R. J. Szabo. D-Branes, Tachyons and K-Homology. Modern Physics Letters A, 17:2297-2315, 2002.

[29] Eugene P. Wigner. Group theory and its application to the quantum mechanics of atomic spectra. Expanded and improved. Translated from the German by J. J. Griffin. Pure and Applied Physics. Vol. 5. Academic Press, New York-London, 1959.

[30] Edward Witten. D-branes and K-theory. J. High Energy Phys., (12):Paper 19, 41 pp. (electronic), 1998. 